\title{
The Impact of Musculoskeletal Injuries Sustained in Road Traffic Crashes on Work-Related Outcomes: A Systematic Review
}

\author{
Elise M. Gane ${ }^{1,2,3,4, *}$, Melanie L. Plinsinga ${ }^{2}$, Charlotte L. Brakenridge ${ }^{2}$ D, Esther J. Smits ${ }^{2}$, Tammy Aplin ${ }^{1,5}$ \\ and Venerina Johnston 1,2 \\ 1 School of Health and Rehabilitation Sciences, The University of Queensland, Brisbane 4072, Australia; \\ t.aplin1@uq.edu.au (T.A.); v.johnston@uq.edu.au (V.J.) \\ 2 Recover Injury Research Centre, The University of Queensland, Brisbane 4066, Australia; \\ m.plinsinga@uq.edu.au (M.L.P.); c.brakenridge@uq.edu.au (C.L.B.); e.smits@uq.edu.au (E.J.S.) \\ 3 Physiotherapy Department, Princess Alexandra Hospital, Brisbane 4102, Australia \\ 4 Centre for Functioning and Health Research, Metro South Health, Brisbane 4102, Australia \\ 5 Allied Health Research Collaborative, The Prince Charles Hospital, Brisbane 4032, Australia \\ * Correspondence: e.gane@uq.edu.au
}

check for updates

Citation: Gane, E.M.; Plinsinga, M.L.; Brakenridge, C.L.; Smits, E.J.; Aplin, T.; Johnston, V. The Impact of

Musculoskeletal Injuries Sustained in Road Traffic Crashes on Work-Related Outcomes: A Systematic Review. Int. J. Environ. Res. Public Health 2021, 18, 11504. https://doi.org/10.3390/ ijerph182111504

Academic Editor: Paul B. Tchounwou

Received: 10 September 2021

Accepted: 25 October 2021

Published: 1 November 2021

Publisher's Note: MDPI stays neutral with regard to jurisdictional claims in published maps and institutional affiliations.

Copyright: (c) 2021 by the authors. Licensee MDPI, Basel, Switzerland. This article is an open access article distributed under the terms and conditions of the Creative Commons Attribution (CC BY) license (https:/ / creativecommons.org/licenses/by/ $4.0 /)$.

\begin{abstract}
Musculoskeletal injuries occur frequently after road traffic crashes (RTCs), and the effect on work participation is not fully understood. The primary aim of this review was to determine the impact of sustaining a musculoskeletal injury during an RTC on the rate of return to work (RTW), sick leave, and other work outcomes. The secondary aim was to determine factors associated with these work-related outcomes. An electronic search of relevant databases to identify observational studies related to work and employment, RTC, and musculoskeletal injuries was conducted. Where possible, outcome data were pooled by follow-up period to answer the primary aim. Fifty-three studies were included in this review, of which 28 were included in meta-analyses. The pooled rate of RTW was $70 \%$ at 1 month, $67 \%$ at 3 months, $76 \%$ at 6 months, $83 \%$ at 12 months, and $70 \%$ at 24 months. Twenty-seven percent of participants took some sick leave by one month follow-up, $13 \%$ by 3 months, $23 \%$ by 6 months, $36 \%$ by 12 months, and $22 \%$ by 24 months. Most of the factors identified as associated with work outcomes were health-related, with some evidence also for sociodemographic factors. While $70 \%$ of people with RTC-related musculoskeletal injury RTW shortly after accident, many still have not RTW two years later.
\end{abstract}

Keywords: musculoskeletal injury; traffic accidents; occupational rehabilitation; return to work

\section{Introduction}

Musculoskeletal injuries occur frequently after road traffic crashes (RTCs) to varying levels of severity. This includes whiplash injury, which is one of the most common injuries [1-3], along with milder injuries such as sprains and strains and more serious injuries such as fractures and dislocations. [4]. A range of negative health outcomes arise from musculoskeletal injuries, including disability and reduced health-related quality of life $[5,6]$. One understudied outcome of sustaining a musculoskeletal injury from an RTC is the impact on paid employment. The nature of the injury may require hospitalisation, which necessitates time off work. Even without hospitalisation, musculoskeletal injuries may leave individuals physically unable to work or with symptoms that interfere with work [7].

Work is important for many reasons, including the promotion of positive health outcomes. There is a recognized link between harm to mental and physical health, and prolonged time off work [8-10]. There is also a link between unemployment and increased mortality rates, as well as increased health service utilisation [11]. In addition, work is considered to be therapeutic — this is a founding principle of work disability management [12]. 
Musculoskeletal disorders is one of the top two diagnoses associated with long-term sick leave, the other being mental disorders [13]. Application of the biopsychosocial model in the successful rehabilitation of individuals with work-related disability is supported by evidence [14] as well as key regulatory authorities and state governments in Australia [15]. The psychological component of the biopsychosocial model is not to be ignored, as previous work has identified expectations of RTW to be significantly predictive of achieving RTW after injury or illness [16], including RTC specifically [17].

Recent work in the field of orthopaedic trauma (not specific to RTCs) suggests that $61 \%$ of injured persons RTW within 2 years post-injury [18] with an average of 100 days of work missed as a result [18]. Having such data regarding workers recovering from RTC-related musculoskeletal injuries would be helpful in assisting injured workers, their employers, and their health professionals understand the potential recovery trajectories and assist in setting rehabilitation and RTW goals. This information could also help to highlight the need for employer and legislated support with regard to RTW. In addition, more evidence is required for the factors associated with work and work-related outcomes in this cohort to inform workplace and employment support systems. A recent systematic review of personal and social factors associated with sustainable RTW (i.e., maintenance of RTW without sick leave) after musculoskeletal disorders and common mental disorders identified five key factors: support from supervisors and co-workers, a positive attitude, self-efficacy, younger age, and higher education levels [19]. Key injury-specific elements such as the influence of compulsory third-party schemes on work-related outcomes warrant further investigation in the post-RTC clinical population.

Delayed RTW and absence from work are not the only work-related outcomes that could be affected by an RTC-related musculoskeletal injury. Work capacity, work ability, and health-related work productivity loss are also key outcomes to consider. Work capacity is often considered to be an individual's capacity to meet the demands of their job, and may require modification of duties or hours of work for example. Work capacity is an important construct when considering the outcomes of RTC, as medical clearance to RTW is often required either by the employer or the insurer [20]. Work ability is defined as the worker's own perceptions of their health and their ability to meet the physical and mental demands of their job [21]. Previous research has shown that musculoskeletal injuries (not specific to RTC) affect workers' occupational readiness and task performance [22], and are an independent predictor of impaired work ability [23]. Health-related work productivity loss is another important work outcome to consider as there are significant costs arising from lost productivity associated with musculoskeletal disorders-missed workdays due to low back pain in the US, for example, lead to US\$28 billion in lost productivity annually [24]. Prior to designing interventions to promote RTW for those with musculoskeletal injuries after RTCs, more needs to be known about the nature of recovery for these individuals across a number of work outcome indicators.

The primary aim of this systematic review is to determine the impact of sustaining a musculoskeletal injury during an RTC on five work-related outcomes: the rate of RTW following injury, the utilisation of sick leave, work capacity, work ability, and health-related work productivity loss. The secondary aim is to determine factors associated with these work-related outcomes in people with musculoskeletal injuries as a result of an RTC.

\section{Materials and Methods}

This review was conducted and reported in compliance with the Preferred Reporting Items for Systematic Review and Meta-Analyses (PRISMA) [25]. This systematic review was registered in the International Prospective Register for Systematic Reviews (PROSPERO) on 14 August 2018 (reference number CRD42018099252). A detailed protocol on the methodological approach has been published [26]. 


\subsection{Search Methods}

An electronic search was conducted in PubMed, EMBASE, Cumulated Index to Nursing and Allied Health Literature (CINAHL), Web of Science, PsycINFO, and Australian Transport Index databases on 2 July 2020. To identify relevant articles, a combination of search terms was used related to work and employment, RTC, observational study design, and musculoskeletal injuries [26]. The search was limited to English studies and all publication years were considered. The search strategy was developed in PubMed and modified for the other databases. The PubMed search strategy can be found in Supplementary Material S1.

\subsection{Eligibility Criteria}

This systematic review included published studies of individuals who sustained musculoskeletal injuries during an RTC on work-related outcomes.

\subsubsection{Population}

Studies were eligible for inclusion if participants were adults who sustained a musculoskeletal injury during an RTC. Studies that investigated outcomes after neurological injuries such as spinal cord injury and traumatic brain injury were excluded, as recent work has already been done in this space $[27,28]$. Other studies were excluded if the same participants had mixed musculoskeletal and neurological injuries (multisystem trauma). If the cohort was a mix of individuals with musculoskeletal injuries and individuals with neurological injuries, the study was included if results were presented separately for those with musculoskeletal injuries.

Adults were the focus of this review, and thus studies with exclusively paediatric cohorts were excluded. However, studies that consisted of a small percentage of adolescent participants were still included if adults remained the majority of the cohort [29-32]. This is a deviation from our published protocol [26]. The authors felt the inclusion of the highly relevant results from these studies added strength to this review, outweighed the disadvantage of deviating from the protocol, and recognized the fact that adolescents can be employed and experience a negative impact on their work if injured.

\subsubsection{Outcome}

Five work-related outcomes were the focus of this review, and included studies needed to report at least one of: (i) RTW rate, (ii) sick leave, (iii) work ability, (iv) work capacity, or (v) health-related work productivity loss. These work-related outcomes could be reported as either a primary or secondary outcome of the study.

\subsubsection{Study Design}

Included studies were observational in design, including cross-sectional, retrospective, and prospective. If participants engaged in rehabilitation programs that were considered 'usual care' or those that occur in 'real world' settings (e.g., participants would have received this treatment regardless of whether they were included in a research study or not), those studies were considered observational and were included in the present review. Studies were excluded if they were randomized controlled trials or case studies, or prepost-intervention designs implemented by the study authors (not usual care). These criteria were applied in order to capture outcomes from participants recovering under 'real world' circumstances, and not experimentally controlled care.

\subsubsection{Language}

Only studies published in English were eligible for this review.

\subsection{Study Selection}

Title and abstract screening and full-text screening was conducted independently by two researchers (divided among authors EG, ES, CB, TA) in Microsoft Excel. Author EG 
screened all title and abstracts, with the second screening conducted by TA (60\%), CB (30\%) and ES (10\%), allocated at random. Full-text screening was equally distributed between EG, TA and ES. Allocation to author for screening occurred in a manner to enable equal crossover amongst the three authors (e.g., 50\% of EG's full texts were also reviewed by ES, and the other $50 \%$ by TA). Discrepancies were resolved by group discussion or by senior author VJ. Reference lists of included studies and relevant systematic reviews identified in the search were reviewed for eligible studies.

\subsection{Data Extraction}

Data extraction was divided evenly amongst the authors (EG, ES, CB, TA, MP) so that each study was examined independently by two authors, with consensus achieved by consultation with the senior author VJ. Allocation for data extraction was similar to fulltext review in that there was approximately equal cross-over amongst authors. Data were extracted into a predetermined Excel form that has been described in detail previously [26]. Authors of included studies were contacted by authors EG or MP if data was incomplete or if data needed to be confirmed.

\subsection{Quality Appraisal}

Study quality was assessed with the National Institutes of Health (NIH) National Heart, Lung, and Blood Institute (NHLBI) Study Quality Assessment Tools for observational cohort and cross-sectional studies, and case-control studies [33]. Co-authors (EG, ES, CB, TA, MP) conducted two independent quality assessments of each result on the same articles on which they conducted data extraction and consensus was reached with the senior author VJ.

\subsection{Analysis}

Inter-rater reliability of the quality assessment tool was examined using kappa $(\mathrm{k})$ statistics (SPSS version 25.0; IBM Corp, Chicago IL). Reliability was considered as slight $(0.00-0.2)$, fair $(0.21-0.4)$, moderate $(0.41-0.6)$, substantial $(0.61-0.8)$ or almost perfect (0.81-1.0) [34].

Meta-analysis was conducted provided that at least two studies reported on the same work outcome including variability estimates. Categorical outcomes (e.g., percentage of people returned to work) were transformed using the Freeman-Tukey double arcsine method [35] and pooled prevalence scores were calculated in Medcalc (MedCalc Software bv Ostend, Belgium). No continuous data were identified as suitable for meta-analysis. Statistical heterogeneity of $\mathrm{I}^{2}=25 \%$ was considered low, $50 \%$ moderate, and $75 \%$ high [36].

Sensitivity analyses were performed to assess the robustness of the outcomes by excluding the studies rated as 'poor' on the quality appraisal tool. Narrative synthesis was used to summarise and explain the conclusions across the studies.

\section{Results}

\subsection{Study Selection}

Figure 1 shows that the electronic search identified 2324 studies. After removing 801 duplicates, 1523 records were screened on title and abstract with 166 included for full-text review. Finally, 53 studies were included in the narrative synthesis and 28 studies were included for meta-analysis. Five pairs of studies $(n=10)$ used (partially) the same participant cohorts [29,30,37-44]; all studies were kept in the narrative review. Of the ten studies, four were included in meta-analyses of data that were not repeated in their paired study [29,39,40,43], and in the case of Gopinath and colleagues [41,42], the 2015 study was used in the meta-analysis of RTW as more detailed data were reported in that study. When multiple articles from the same study reported the same work-related outcome measure, the article with the highest number of participants was used in the meta-analysis and the other articles were excluded from the meta-analysis to avoid overlap in participants $[38,39,42,44]$. 


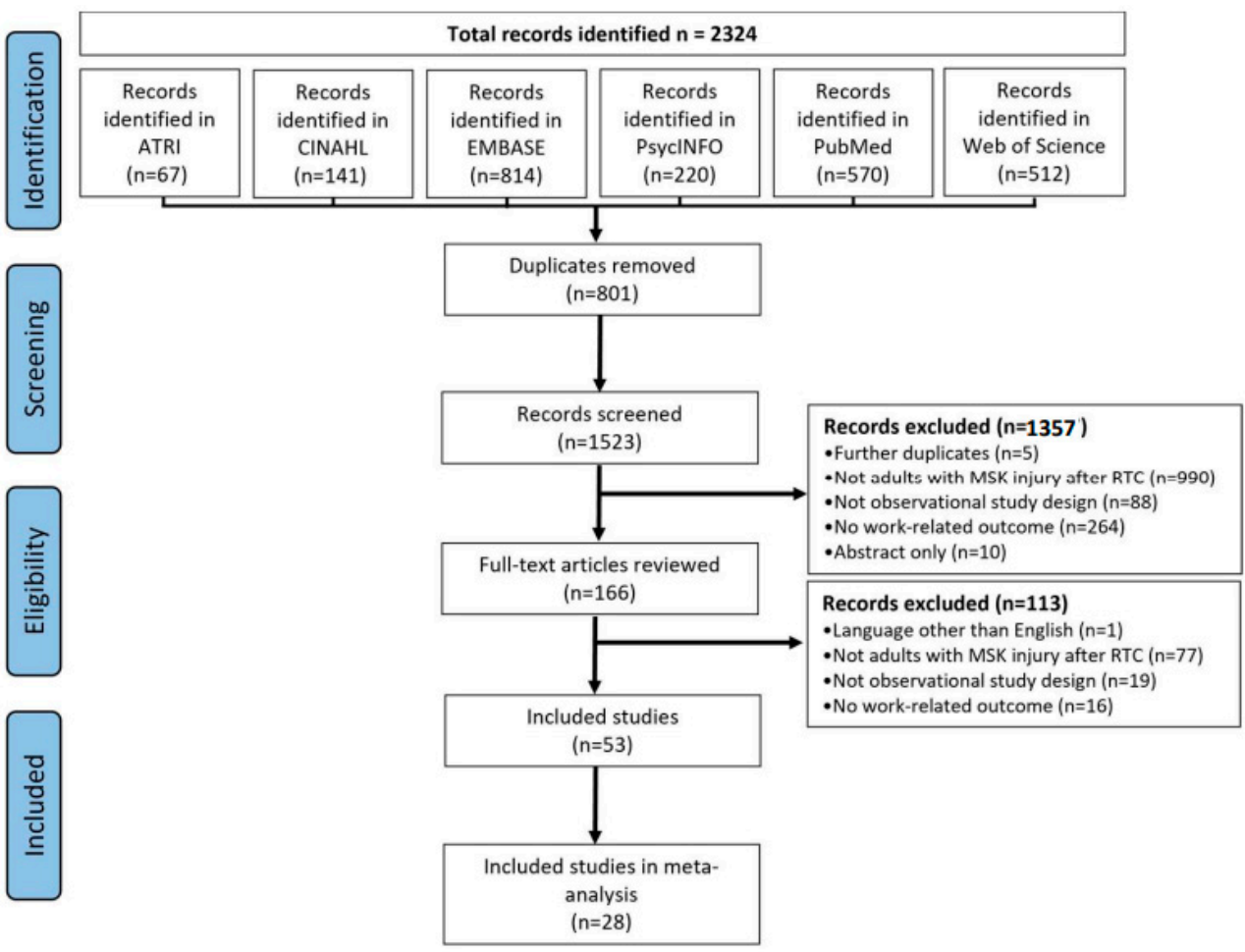

Figure 1. PRISMA flowchart.

\subsection{Study Characteristics}

The main characteristics of the included studies are described in Table 1. Of the 53 studies, 33 had a prospective cohort design, 13 were retrospective studies, and 7 studies had a cross-sectional design. The sample size varied from 11 [45] to 32,970 [30] participants. Most studies were conducted in Europe $(n=22,42 \%)$, followed by Australia $(n=17,30 \%)$, North America $(n=10,19 \%)$, South America $(n=2$ Brazil and Chile, $4 \%)$, Africa $(n=1$ Uganda, $2 \%$ ) and the Middle East ( $n=1$ Israel, $2 \%$ ). Participants were sampled through various means including insurance companies and the general public (Table 1).

Of the $n=48$ participant cohorts across the $n=53$ included studies, most investigated patients with whiplash injuries $(29 / 48,60 \%)$, various musculoskeletal injuries (e.g., low back pain, neck pain, lower limb and hip fractures) $(16 / 48,34 \%)$, and mixed cohorts consisting of whiplash and other musculoskeletal injuries $(3 / 48,6 \%)$. The follow-up assessments from time of injury ranged from 3 days [46] to 8.5 years [47]. 
Table 1. Study characteristics of included studies.

\begin{tabular}{|c|c|c|c|c|c|c|c|c|}
\hline $\begin{array}{l}\text { First Author } \\
\text { and Year }\end{array}$ & Country & $\begin{array}{l}\text { Study Design } \\
\text { and Size }\end{array}$ & Study Setting & Age (Years) & $\begin{array}{l}\text { Sex (Female } \\
\quad N(\%))\end{array}$ & Injury Type & $\begin{array}{c}\text { Assessment Time } \\
\text { (Baseline, Follow Up) }\end{array}$ & Specific Outcome Measures \\
\hline $\begin{array}{c}\text { Ackland } 2013 \\
\text { [48] }\end{array}$ & Australia & $\begin{array}{l}\text { Prospective } \\
\text { cohort, } 178\end{array}$ & Hospital based & Median 35 (IQR 25-48) & $72(44.0 \%)$ & Neck pain & $\begin{array}{l}\text { Baseline, after discharge, } \\
\qquad 6 \mathrm{~m}, 12 \mathrm{~m}\end{array}$ & $\begin{array}{c}\text { RTW: return to full duties, } \\
\text { categorical }(n, \%) \\
\text { Work capacity: period of } \\
\text { modified work, categorical }(n, \%) \\
\text { Work ability: categorical }(n, \%)\end{array}$ \\
\hline $\begin{array}{l}\text { Barbosa } 2014 \\
\quad[49]\end{array}$ & Brazil & $\begin{array}{l}\text { Cross-sectional, } \\
210\end{array}$ & General public & $\begin{array}{c}\text { Aged between } 18 \text { and } 35 \\
\text { years } n=59 ; \text { aged between } 36 \\
\text { and } 45 \text { years } n=64 \text {; age }>45 \\
\text { years } n=42\end{array}$ & Men only & Facial trauma & One time & $\begin{array}{c}\text { Sick leave: categorical }(n, \%) \\
\text { Productivity loss: absenteeism, } \\
\text { categorical }(n, \%)\end{array}$ \\
\hline $\begin{array}{l}\text { Berecki-Gisolf } \\
2013[7]\end{array}$ & Australia & $\begin{array}{l}\text { Retrospective } \\
\text { cohort, } 5970\end{array}$ & $\begin{array}{l}\text { Transport } \\
\text { Accident } \\
\text { Commission }\end{array}$ & $\begin{array}{l}24.0 \% \text { aged between } 35 \\
\text { and } 44\end{array}$ & $2208(37.0 \%)$ & MSK or orthopaedic & $\begin{array}{l}\text { Once (17 months after } \\
\text { accident) }\end{array}$ & $\begin{array}{c}\text { Sick leave: compensated days off } \\
\text { work, categorical }(\%)\end{array}$ \\
\hline $\begin{array}{c}\text { Biering- } \\
\text { Sorensen } 2014 \\
{[50]}\end{array}$ & Denmark & $\begin{array}{c}\text { Prospective } \\
\text { cohort, } 7780 \text { (104 } \\
\text { WAD; } 3204 \text { other } \\
\text { MSK) }\end{array}$ & Municipalities & WAD mean 36; MSK mean 42 & $\begin{array}{l}\text { WAD } 75.0 \% \\
\text { MSK } 52.0 \%\end{array}$ & $\begin{array}{l}\text { Whiplash and other } \\
\text { MSK pains }\end{array}$ & $\begin{array}{c}\text { At } 26 \text { weeks, } 1 \text { year, } 2 \\
\text { years, } 3 \text { years }\end{array}$ & $\begin{array}{l}\text { RTW: categorical }(n, \%) \\
\text { Sick leave: number of } \\
\text { participants sick listed, } \\
\text { categorical }(n)\end{array}$ \\
\hline $\begin{array}{l}\text { Borchgrevink } \\
1996[47]\end{array}$ & Norway & $\begin{array}{l}\text { Retrospective } \\
\text { cohort, } 426\end{array}$ & $\mathrm{ED}$ & $\begin{array}{c}\text { Men median } 37 \text { (IQR 28-47); } \\
\text { women } 36 \text { (IQR 26-48) }\end{array}$ & $\begin{array}{l}\text { Men } 174(41.0 \%) \\
\text { women } 252 \\
(59.0 \%)\end{array}$ & Whiplash & $\begin{array}{l}\text { Prior to injury, follow up } \\
\text { after injury (between } 2.5 \\
\text { and } 8-5 \text { years) }\end{array}$ & $\begin{array}{l}\text { RTW: categorical }(n) \\
\text { Sick leave: registration for sick } \\
\text { leave, categorical }(n, \%)\end{array}$ \\
\hline Brison 2000 [51] & Canada & $\begin{array}{l}\text { Prospective } \\
\text { cohort, } 353\end{array}$ & EDs & $\begin{array}{c}\text { Aged } 18-30 \text { year } n=140 \\
(39.7 \%) ; \text { aged } 31-50 \text { years } \\
n=150(42.5 \%) ; \text { aged } 51-70 \\
\text { years } n=63(17.8 \%)\end{array}$ & $224(63.5 \%)$ & Whiplash & $\begin{array}{c}\text { Baseline, 1, 2, 3, 6, 9, 12, } \\
\text { 18, } 24 \text { months }\end{array}$ & $\begin{array}{l}\text { Sick leave: missing }<1 \text { week of } \\
\text { work, categorical (\%); days } \\
\text { missed work, continuous (mean) } \\
\text { Work capacity: number of } \\
\text { participants that modified work } \\
\text { activities, categorical (\%) }\end{array}$ \\
\hline $\begin{array}{c}\text { Buitenhuis } 2009 \\
\text { [52] }\end{array}$ & $\begin{array}{l}\text { The } \\
\text { Netherlands }\end{array}$ & $\begin{array}{l}\text { Prospective } \\
\text { cohort, } 879\end{array}$ & $\begin{array}{l}\text { Insurance } \\
\text { company }\end{array}$ & Mean 36 (SD 12) & $539(61.3 \%)$ & Whiplash & Baseline, 6, 12 months & $\begin{array}{l}\text { RTW: number of participants } \\
\text { with no work or paid } \\
\text { employment, categorical }(n, \%) \text {; } \\
\text { working hours per week, } \\
\text { continuous (mean, SD) } \\
\text { Sick leave: people on work } \\
\text { disability, categorical }(n, \%) \text {. }\end{array}$ \\
\hline $\begin{array}{c}\text { Bunketorp } 2002 \\
{[53]}\end{array}$ & Sweden & $\begin{array}{l}\text { Retrospective } \\
\text { cohort, } 108\end{array}$ & $\begin{array}{l}\text { EDs in } 2 \text { main } \\
\text { hospitals }\end{array}$ & Mean 52 (SD 13) & $66(61.0 \%)$ & Whiplash & $\begin{array}{l}\text { Once (17 years after } \\
\text { injury) }\end{array}$ & $\begin{array}{c}\text { Sick leave: number of participant } \\
\text { on sick leave, work disability, or } \\
\text { early retirement, categorical (\%) } \\
\text { Work ability: assigned medical } \\
\text { disability, categorical }(n, \%)\end{array}$ \\
\hline Bylund 1998 [54] & Sweden & $\begin{array}{l}\text { Prospective } \\
\text { cohort, } 255\end{array}$ & General public & $\begin{array}{l}\text { Women mean } 33 ; \text { men } \\
\text { mean } 31\end{array}$ & $132(52.0 \%)$ & $\begin{array}{l}\text { Cervical strain/ } \\
\text { fracture }\end{array}$ & $\begin{array}{c}\text { During the 2-year period } \\
\text { from Jan 1,1990 to Dec } \\
\text { 31, } 1991\end{array}$ & $\begin{array}{c}\text { Sick leave: categorical }(n, \%) \text { per } \\
\text { collusion mechanism; total sick } \\
\text { leave days. }\end{array}$ \\
\hline
\end{tabular}


Table 1. Cont




Table 1. Cont.

\begin{tabular}{|c|c|c|c|c|c|c|c|c|}
\hline $\begin{array}{l}\text { First Author } \\
\text { and Year }\end{array}$ & Country & $\begin{array}{l}\text { Study Design } \\
\text { and Size }\end{array}$ & Study Setting & Age (Years) & Sex (Female $N(\%)$ ) & Injury Type & $\begin{array}{l}\text { Assessment } \\
\text { Time (Baseline, } \\
\text { Follow Up) }\end{array}$ & Specific Outcome Measures \\
\hline Gray ${ }^{4} 2018$ [29] & Australia & $\begin{array}{l}\text { Retrospective } \\
\text { cohort, } 24311\end{array}$ & $\begin{array}{l}\text { Transport } \\
\text { Accident } \\
\text { Commission }\end{array}$ & $\begin{array}{l}22.0 \% \text { aged } 15-24 ; 25.1 \% \text { aged } \\
25-34 ; 22.7 \% \text { aged } 35-44 ; 19.3 \% \\
\text { aged } 45-54 ; 11.0 \% \text { aged } 55-70 .\end{array}$ & $36.9 \%$ & Various MSK injuries & NA & $\begin{array}{l}\text { RTW: failed and sustained RTW, } \\
\text { categorical }(n, \%)\end{array}$ \\
\hline Gray $^{4} 2018$ [30] & Australia & $\begin{array}{l}\text { Retrospective } \\
\text { cohort, } 32970\end{array}$ & $\begin{array}{l}\text { Transport } \\
\text { Accident } \\
\text { Commission }\end{array}$ & $\begin{array}{l}22.0 \% \text { aged } 15-24 ; 25.1 \% \text { aged } \\
25-34 ; 22.7 \% \text { aged } 35-44 ; 19.1 \% \\
\text { aged } 45-54 ; 11.0 \% \text { aged } 55-70 .\end{array}$ & $36.0 \%$ & Various MSK injuries & NA & $\begin{array}{c}\text { Work capacity: Gradual RTW } \\
\text { pathway, categorical }(n ., \%) \text { and } \\
\text { days (median, IQR) }\end{array}$ \\
\hline Guest 2017 [58] & Australia & $\begin{array}{c}\text { Retrospective } \\
\text { cohort, 6341 } \\
\text { (MSK } n=5734 ; \\
\text { MSK + } \\
\text { psychological } \\
\text { distress } \\
\text { (MSKPD) } \\
n=607)\end{array}$ & $\begin{array}{l}\text { SIRA Personal } \\
\text { Injury Register }\end{array}$ & $\begin{array}{l}\text { MSK group mean } 43 \text { (SD 16); } \\
\text { MSKPD group mean } 44 \text { (SD 16) }\end{array}$ & $\begin{array}{l}\text { MSK group } 3317 \\
(58.0 \%) ; \text { MSKPD } \\
\text { group } 395(65.0 \%)\end{array}$ & Various MSK injuries & Once & $\begin{array}{l}\text { Productivity loss: economic loss } \\
\text { claim, categorical }(n, \%)\end{array}$ \\
\hline Gun 2005 [59] & Australia & $\begin{array}{l}\text { Prospective } \\
\text { cohort, } 135\end{array}$ & $\begin{array}{l}\text { EDs, medical } \\
\text { and physio } \\
\text { practices }\end{array}$ & Mean 36 (SD 15) & $98(83.0 \%)$ & Whiplash & $\begin{array}{l}\text { Baseline, } \\
6 \text { weeks, } \\
12 \text { months }\end{array}$ & RTW: categorical $(n, \%)$ \\
\hline $\begin{array}{l}\text { Herrström } 2000 \\
{[60]}\end{array}$ & Sweden & $\begin{array}{l}\text { Prospective } \\
\text { cohort, } 158\end{array}$ & $\begin{array}{l}\text { Health } \\
\text { care-based } \\
\text { register }\end{array}$ & $\begin{array}{l}\text { Range 10-77; women median 30; } \\
\text { men median } 35\end{array}$ & $\begin{array}{c}74 / 125(59.0 \%) \\
\text { (reported for } \\
12 \text { month data } \\
n=125)\end{array}$ & Whiplash & $\begin{array}{l}\text { Baseline, } \\
12 \text { months }\end{array}$ & $\begin{array}{c}\text { Sick leave: reported sick leave, } \\
\text { categorical }(n, \%) \text {; duration sick } \\
\text { leave (mean, range) }\end{array}$ \\
\hline $\begin{array}{l}\text { Hildingsson } \\
1990[61]\end{array}$ & Sweden & $\begin{array}{l}\text { Prospective } \\
\text { cohort, } 93\end{array}$ & University based & Median 31 (range 17-67) & $53(57.0 \%)$ & Whiplash & $\begin{array}{l}\text { Baseline, follow } \\
\text { up (mean } \\
25 \text { months) }\end{array}$ & $\begin{array}{l}\text { Sick leave: reported sick leave, } \\
\text { categorical }(n) \\
\text { Work capacity: changes in work } \\
\text { status, categorical }(n)\end{array}$ \\
\hline Holm 1999 [62] & Sweden & $\begin{array}{l}\text { Cross-sectional, } \\
\quad 4121\end{array}$ & $\begin{array}{l}\text { Road Traffic } \\
\text { Injury } \\
\text { Commission }\end{array}$ & $\begin{array}{l}\text { WAD 1989: partial or full work } \\
\text { disability }(n=172) \text { mean } 47 \text { (SD } \\
\text { 10); no work disability }(n=106) \\
\text { mean } 39 \text { (SD 11) } \\
\text { WAD 1994: partial or full work } \\
\text { disability }(n=417) \text { mean } 43 \text { (SD } \\
\text { 10); no work disability }(n=277) \\
\text { mean } 37 \text { (SD 11) }\end{array}$ & $\begin{array}{l}\text { WAD 1989: partial or } \\
\text { full work disability } \\
\text { 100/172 (57.6\%); no } \\
\text { work disability } \\
71 / 106(67.0 \%) \\
\\
\text { WAD 1994: partial or } \\
\text { full work disability } \\
\text { 256/417 (61.4\%); no } \\
\text { work disability } \\
\text { 156/277 (56.3\%) }\end{array}$ & Whiplash & Once & $\begin{array}{c}\text { RTW: return to full work capacity, } \\
\text { categorical (\%) } \\
\text { Work capacity: partial and full } \\
\text { work disability, categorical }(n, \%)\end{array}$ \\
\hline
\end{tabular}


Table 1. Cont.

\begin{tabular}{|c|c|c|c|c|c|c|c|c|}
\hline $\begin{array}{l}\text { First Author } \\
\text { and Year }\end{array}$ & Country & $\begin{array}{l}\text { Study Design } \\
\text { and Size }\end{array}$ & Study Setting & Age (Years) & $\begin{array}{l}\text { Sex (Female } \\
\quad N(\%))\end{array}$ & Injury Type & $\begin{array}{c}\text { Assessment Time } \\
\text { (Baseline, Follow Up) }\end{array}$ & Specific Outcome Measures \\
\hline Hours 2014 [31] & France & $\begin{array}{l}\text { Prospective } \\
\text { cohort, } 253\end{array}$ & $\begin{array}{l}\text { Registry of Road } \\
\text { Crash Trauma }\end{array}$ & 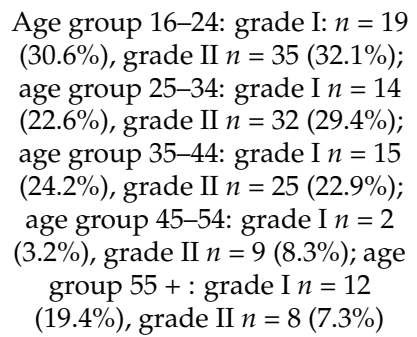 & $157(62.0 \%)$ & Whiplash & Baseline, 12 months & $\begin{array}{l}\text { RTW: participants that did } \\
\text { not RTW, categorical }(\%) \\
\text { Sick leave: categorical }(n, \%) \\
\text { and (median, IQR). }\end{array}$ \\
\hline Hoving 2003 [63] & Australia & $\begin{array}{l}\text { Cross-sectional, } \\
71\end{array}$ & $\begin{array}{l}\text { Physio, GP, } \\
\text { rheumatology } \\
\text { clinics }\end{array}$ & Mean 40 (SD 14) & $59(83.1 \%)$ & Whiplash & Once & $\begin{array}{c}\text { Work ability: Neck Disability } \\
\text { Index-Work Item mean and } \\
\text { The Northwick Park Neck } \\
\text { Pain Questionnaire- } \\
\text { Work/housework, } \\
\text { continuous (mean, SD); } \\
\text { problem elicitation technique } \\
\text { (PET)—work for wages, } \\
\text { categorical }(n, \%)\end{array}$ \\
\hline Kasch 2001 [64] & Denmark & $\begin{array}{l}\text { Prospective } \\
\text { cohort, } 141\end{array}$ & General public & Mean 36 (SD 11); & $74(52.0 \%)$ & Whiplash & $\begin{array}{c}\text { Baseline, } 1 \text { week, } 1,3,6, \\
12 \text { months }\end{array}$ & $\begin{array}{l}\text { RTW: participants that did } \\
\text { not RTW, categorical }(n, \%) \\
\text { Work capacity: returned to } \\
\text { modified job functions, } \\
\text { categorical }(\%)\end{array}$ \\
\hline Kasch 2011 [65] & Denmark & $\begin{array}{l}\text { Prospective } \\
\text { cohort, } 141\end{array}$ & EDs & NR & NR & Whiplash & $\begin{array}{l}\text { At } 1 \text { week, } 1,3,6, \\
12 \text { months }\end{array}$ & RTW: categorical $(n, \%)$ \\
\hline Kasch 2019 [66] & Denmark & $\begin{array}{l}\text { Prospective } \\
\text { cohort, } 143\end{array}$ & EDs & Mean 35.8 (SD 10.5) & $75(52.0 \%)$ & Whiplash & At 1 week, 6, 12 months & $\begin{array}{l}\text { Work capacity: participants } \\
\text { 'recovered' }(n, \%)\end{array}$ \\
\hline Kinzel 2006 [45] & Australia & $\begin{array}{l}\text { Prospective } \\
\text { cohort, } 11\end{array}$ & Hospital based & Mean 34 (range 16-67) & $1(9.0 \%)$ & $\begin{array}{l}\text { Upper limb MSK } \\
\text { injuries }\end{array}$ & $\begin{array}{l}\text { Baseline, regular } \\
\text { outpatient appointments } \\
\text { up to } 12 \text { months }\end{array}$ & RTW: categorical $(n)$ \\
\hline Krogh 2018 [67] & Denmark & $\begin{array}{l}\text { Prospective } \\
\text { cohort, } 141\end{array}$ & EDs & Mean 36 (SD 11) & $74(52.0 \%)$ & Whiplash & $\begin{array}{l}\text { Baseline, 1, 3, 6, } \\
\quad 12 \text { months }\end{array}$ & $\begin{array}{c}\text { Work capacity: participants' } \\
\text { recovery status, categorical } \\
(n, \%)\end{array}$ \\
\hline $\begin{array}{l}\text { Leth-Petersen } \\
2009[68]\end{array}$ & Denmark & $\begin{array}{l}\text { Retrospective } \\
\text { cohort, } 1203\end{array}$ & $\begin{array}{l}\text { Records of } \\
\text { National Board } \\
\text { of Industrial } \\
\text { Injuries }\end{array}$ & $\begin{array}{c}\text { Women-comp mean } 36 \text { (SD 9); } \\
\text { women-noncomp } 36 \text { (SD 9); } \\
\text { men-comp } 35 \text { (SD 8); } \\
\text { men-noncomp } 36 \text { (SD 9) }\end{array}$ & $844(70.0 \%)$ & Whiplash & Retrospective data & $\begin{array}{l}\text { Productivity loss: percentage } \\
\text { of lost earnings capacity, } \\
\text { categorical }(n, \%)\end{array}$ \\
\hline
\end{tabular}


Table 1. Cont.

\begin{tabular}{|c|c|c|c|c|c|c|c|c|}
\hline $\begin{array}{l}\text { First Author } \\
\text { and Year }\end{array}$ & Country & $\begin{array}{l}\text { Study Design } \\
\text { and Size }\end{array}$ & Study Setting & Age (Years) & $\begin{array}{c}\text { Sex (Female } \\
N(\%))\end{array}$ & Injury Type & $\begin{array}{c}\text { Assessment Time } \\
\text { (Baseline, Follow Up) }\end{array}$ & Specific Outcome Measures \\
\hline $\begin{array}{l}\text { Mankovsky- } \\
\text { Arnold 2017 } \\
\text { [69] }\end{array}$ & Canada & $\begin{array}{l}\text { Cross-sectional, } \\
105\end{array}$ & $\begin{array}{l}\text { Rehabilitation } \\
\text { clinics }\end{array}$ & $\begin{array}{c}\text { Overall mean } 37 \text { (range } \\
\text { 17-57); men mean } 38 \text { (SD 9); } \\
\text { women } 36 \text { (SD 11). }\end{array}$ & $49(47.0 \%)$ & Whiplash & Once & $\begin{array}{l}\text { RTW: employment status, } \\
\text { categorical }(n, \%)\end{array}$ \\
\hline $\begin{array}{l}\text { Miettinen }{ }^{5} 2004 \\
{[43]}\end{array}$ & Finland & $\begin{array}{l}\text { Prospective } \\
\text { cohort, } 182\end{array}$ & $\begin{array}{l}\text { Insurance } \\
\text { companies }\end{array}$ & $\begin{array}{l}\text { Overall mean } 42 ; \text { men } 45 \\
\text { (SD 15); women } 40 \text { (SD 14) }\end{array}$ & $117(64.3 \%)$ & Whiplash & Baseline, 12 months & $\begin{array}{c}\text { Sick leave: (length of) sick leave, } \\
\text { categorical }(n, \%)\end{array}$ \\
\hline$\underset{[44]}{\text { Miettinen }}{ }^{5} 2002$ & Finland & $\begin{array}{l}\text { Prospective } \\
\text { cohort, } 201\end{array}$ & $\begin{array}{l}\text { Insurance } \\
\text { companies }\end{array}$ & Mean 42 & NR & Whiplash & Baseline, 12 months & $\begin{array}{c}\text { Sick leave: (length of) sick leave, } \\
\text { categorical }(n, \%)\end{array}$ \\
\hline Munjin 2011 [70] & Chile & $\begin{array}{l}\text { Retrospective } \\
\text { cohort, } 46\end{array}$ & Hospital based & Mean 49 (range 16-70) & $37(80.4 \%)$ & $\begin{array}{l}\text { Spine fracture (no } \\
\text { neurologic } \\
\text { impairment) }\end{array}$ & Retrospective data & $\begin{array}{c}\text { Sick leave: leave of absence, } \\
\text { continuous (average, median, } \\
\text { range) }\end{array}$ \\
\hline $\begin{array}{c}\text { Myrtveit } 2015 \\
{[71]}\end{array}$ & Denmark & $\begin{array}{l}\text { Prospective } \\
\text { cohort, } 740\end{array}$ & $\begin{array}{l}\text { Clinics in } \\
\text { University } \\
\text { Hospitals }\end{array}$ & Mean 35 (SD 11) & $475(64.1 \%)$ & Whiplash & Baseline, 12 months & $\begin{array}{l}\text { Work ability: participants that } \\
\text { reported reduced work capability, } \\
\text { categorical }(n, \%)\end{array}$ \\
\hline $\begin{array}{c}\text { Nguyen } 2019 \\
{[32]}\end{array}$ & Australia & $\begin{array}{l}\text { Prospective } \\
\text { cohort, } 498\end{array}$ & $\begin{array}{c}\text { EDs, rural health } \\
\text { services, } \\
\text { primary care and } \\
\text { the NSW State } \\
\text { Insurance } \\
\text { Regulatory } \\
\text { Authority- } \\
\text { Personal Injury } \\
\text { Registry, Claims } \\
\text { Advisory } \\
\text { Service. }\end{array}$ & $\begin{array}{c}\text { Neck injuries: mean } 40.2 \\
\text { (SD 16) } \\
\text { Lower back injuries: mean } \\
35.7 \text { (SD 17) } \\
\text { Lower limb injuries: mean } \\
38.9 \text { (SD 15) }\end{array}$ & $\begin{array}{c}\text { Neck injuries: } \\
107(64.5 \%) \\
\text { Lower back } \\
\text { injuries: } 31 \\
(39.7 \%) \\
\text { Lower limb } \\
\text { injuries: } 78 \\
(30.7 \%)\end{array}$ & Various MSK injuries & Baseline, 6 months & RTW: categorical $(n, \%)$ \\
\hline O'Hara 2018 [72] & Uganda & $\begin{array}{l}\text { Prospective } \\
\text { cohort, } 57\end{array}$ & Hospital based & Median 34 (IQR 27-45) & $9(11.1 \%)$ & $\begin{array}{l}\text { Isolated } \\
\text { tibial/femoral } \\
\text { fracture }\end{array}$ & $\begin{array}{c}\text { Baseline (within } 48 \text { hrs of } \\
\text { hospital admission), } 6, \\
12,24 \text { months }\end{array}$ & $\begin{array}{l}\text { RTW: categorical }(n, \%) \text {; time to } \\
\text { RTW, continuous (mean, } 95 \% \mathrm{CI} \text { ) } \\
\text { Productivity loss: reduction in } \\
\text { monthly income, continuous } \\
\text { (mean difference, } 95 \% \mathrm{CI} \text { ); debts, } \\
\text { continuous (mean, 95\%CI) }\end{array}$ \\
\hline Pieske 2010 [73] & Germany & $\begin{array}{l}\text { Prospective } \\
\text { cohort, } 81\end{array}$ & Hospital based & $\begin{array}{c}\text { Mean } 33.0 \text { (SD 12.0), range } \\
\text { 18-74 }\end{array}$ & $45(55.6 \%)$ & Whiplash & Baseline, 1, 3, 6 months & $\begin{array}{c}\text { RTW: inability to work, } \\
\text { categorical }(n, \%) \\
\text { Sick leave: duration of not being } \\
\text { at work, categorical }(n, \%)\end{array}$ \\
\hline Prang 2015 [74] & Australia & $\begin{array}{l}\text { Cross-sectional, } \\
1649\end{array}$ & General public & Mean 44 (SD 15). & $685(41.5 \%)$ & Various MSK injuries & Once & RTW: categorical $(n, \%)$ \\
\hline
\end{tabular}


Table 1. Cont.

\begin{tabular}{|c|c|c|c|c|c|c|c|c|}
\hline $\begin{array}{l}\text { First Author } \\
\text { and Year }\end{array}$ & Country & Study Design and Size & Study Setting & Age (Years) & $\begin{array}{l}\text { Sex (Female } \\
\quad N(\%))\end{array}$ & Injury Type & $\begin{array}{c}\text { Assessment Time } \\
\text { (Baseline, Follow Up) }\end{array}$ & Specific Outcome Measures \\
\hline Ratzon 2015 [75] & Israel & $\begin{array}{c}\text { Cross-sectional, } 123 \\
\text { (Whiplash } n=76 \text {; Hip } \\
n=47 \text { ) }\end{array}$ & $\begin{array}{c}\text { Outpatient } \\
\text { clinics and } \\
\text { hospital }\end{array}$ & $\begin{array}{l}\text { Whiplash mean } 33 \text { (SD } \\
\text { 11.8); hip } 38 \text { (12) }\end{array}$ & $\begin{array}{l}\text { Whiplash } 40 \\
(52.0 \%) ; \text { hip } 12 \\
(25.5 \%)\end{array}$ & $\begin{array}{l}\text { Whiplash and } \\
\text { hip injury }\end{array}$ & Once & $\begin{array}{l}\text { Work ability: degree of disability } \\
\text { (based on medical files), } \\
\text { categorical }(n, \%)\end{array}$ \\
\hline $\begin{array}{c}\text { Rebbeck } 2006 \\
{[76]}\end{array}$ & Australia & Prospective cohort, 114 & $\begin{array}{l}\text { Insurance } \\
\text { databases }\end{array}$ & Mean 39.4 (SE 1.3) & $\begin{array}{c}\text { Gender female } \\
n=79(69.3 \%) \\
\text { male } n=35 \\
\quad(30.7 \%)\end{array}$ & Whiplash & $\begin{array}{l}\text { Baseline (at } 3 \text { months), } \\
6 \text { months, } 2 \text { years }\end{array}$ & $\begin{array}{c}\text { Sick leave: participants taking } \\
\text { days off work, categorical }(n, \%) ; \\
\text { number of days taken off work, } \\
\text { continuous (median, IQR) }\end{array}$ \\
\hline $\begin{array}{c}\text { Rosenthal } 1979 \\
\text { [77] }\end{array}$ & USA & Retrospective cohort, 43 & $\begin{array}{l}\text { University } \\
\text { hospital }\end{array}$ & NR & NR & $\begin{array}{l}\text { Hip frac- } \\
\text { ture/dislocation }\end{array}$ & Retrospective data & $\begin{array}{c}\text { RTW: not returning to work, } \\
\text { categorical }(n, \%) \\
\text { RTW: categorical }(\%)\end{array}$ \\
\hline $\begin{array}{l}\text { Sarrami } 2016 \\
\quad[78]\end{array}$ & Australia & Retrospective cohort, 90 & $\begin{array}{l}\text { SIRA Personal } \\
\text { Injury Register }\end{array}$ & $\begin{array}{c}\text { Mean } 46 \text { (SD 12), range } \\
\text { 23-73 }\end{array}$ & $48(53.0 \%)$ & Spine surgery & Retrospective data & $\begin{array}{l}\text { Work capacity: return to } \\
\text { pre-injury duties, categorical (\%) }\end{array}$ \\
\hline $\begin{array}{c}\text { Schreiber } 2009 \\
\text { [79] }\end{array}$ & USA & Prospective cohort, 38 & $\begin{array}{l}\text { Private } \\
\text { chiropractic } \\
\text { offices }\end{array}$ & Mean 37 & $\begin{array}{l}\text { "male to female } \\
\text { ratio nearly 1:1" }\end{array}$ & Whiplash & Once & $\begin{array}{c}\text { RTW: employment, categorical } \\
(\%) \\
\text { Work ability: decreased work } \\
\text { function, categorical }(n)\end{array}$ \\
\hline Scuderi 2005 [80] & USA & $\begin{array}{l}\text { Prospective cohort, } 270 \\
\text { (Workers Compensation } \\
\text { (WC) group } n=54 ; \\
\text { Personal Injury (PI) } \\
\text { group } n=216 \text { ) }\end{array}$ & $\begin{array}{l}\text { Workmen's } \\
\text { Compensation } \\
\text { System }\end{array}$ & $\begin{array}{l}\text { WC group mean } 43 \\
\text { (range 25-62); PI group } \\
\text { mean } 35 \text { (range 18-68) }\end{array}$ & $\begin{array}{l}\text { WC group } 20 \\
(37.0 \%) ; \text { PI group } \\
112(51.9 \%)\end{array}$ & Neck pain & $\begin{array}{l}\text { Baseline (referral to } \\
\text { surgeon), follow ups } \\
\text { until considered RTW/ } \\
\text { reaching max } \\
\text { improvement/ lost to } \\
\text { follow up after } 2 \text { years }\end{array}$ & $\begin{array}{l}\text { RTW: unable to RTW, categorical } \\
(n) \\
\text { Sick leave: lost days of work, } \\
\text { continuous (total, mean) }\end{array}$ \\
\hline Smed 1997 [81] & Denmark & Prospective cohort, 29 & $\begin{array}{l}\text { University } \\
\text { hospital }\end{array}$ & Median 33 (range 22-56) & $17(58.6 \%)$ & Whiplash & 1 month post-injury & Sick leave: categorical $(n)$ \\
\hline $\begin{array}{c}\text { Swartzman } 1996 \\
{[82]}\end{array}$ & Canada & $\begin{array}{c}\text { Retrospective cohort, } 62 \\
\quad(\text { litigants } n=41 \\
\text { post-litigants } n=21)\end{array}$ & $\begin{array}{l}\text { Private practice, } \\
\text { University } \\
\text { hospital }\end{array}$ & $\begin{array}{l}\text { Litigants mean } 38 ; \\
\text { post-litigants mean } 39\end{array}$ & $\begin{array}{c}\text { Litigants } 31 \\
(76.0 \%) ; \\
\text { post-litigant } 17 \\
\quad(81.0 \%)\end{array}$ & Whiplash & Once & $\begin{array}{l}\text { RTW: employment status, } \\
\text { categorical; number of hours per } \\
\text { week employed outside the } \\
\text { home, continuous (mean) }\end{array}$ \\
\hline Virani 2001 [83] & Canada & $\begin{array}{c}\text { Cross-sectional, } 356 \\
\text { (physicians } n=149 \\
\text { nonphysicians } n=207 \text { ) }\end{array}$ & $\begin{array}{l}\text { University } \\
\text { hospital }\end{array}$ & $\begin{array}{c}\text { Physicians mean } 46 ; \\
\text { nonphysicians mean } 40\end{array}$ & NR & Whiplash & Once & $\begin{array}{l}\text { Sick leave: time off, categorical } \\
(\%)\end{array}$ \\
\hline Vos 2008 [84] & $\begin{array}{l}\text { The } \\
\text { Netherlands }\end{array}$ & Prospective cohort, 42 & GPs & Mean 35 & NR & $\begin{array}{l}\text { Neck pain and } \\
\text { whiplash }\end{array}$ & Baseline, 6, 12, 26, 52 & Sick leave: categorical (\%) \\
\hline
\end{tabular}

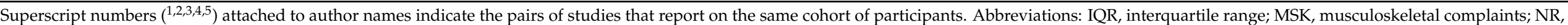
not reported; RTW, return to work; SE, standard error; WAD, whiplash-associated disorder. 
Four studies were included in which adolescents were included in the study cohort [29-32]. Two studies shared the same cohort, $22 \%$ of which was aged $15-24$ years [29,30]. One study presented a cohort in which $32 \%$ of people with whiplash were aged 16-24 years [31]. One study included participants aged at least 17 years but the mean (SD) age was between 35.7 (17) and 40.2 (16) years depending on injury location [32]. Specific details concerning the exact number of participants who were aged $<18$ years could not be ascertained from the authors. Overall, adolescents only consisted of a small portion of the total study populations, and as such these studies were not excluded from the review [32].

\subsection{Quality Appraisal}

Inter-rater agreement for quality appraisal of individual studies was substantial $(\kappa=0.70, p<0.001)$ with $602 / 742$ agreements [34].

Results of the quality appraisal of the individual studies are presented in Table 2. Twenty-one studies were of good quality $(40 \%), 16$ studies of fair quality $(30 \%)$ and 16 studies of poor quality $(30 \%)$. The majority of studies clearly defined the research question ( $n=45,85 \%)$, clearly specified and defined the study population $(n=48,91 \%)$, selected or recruited participants from same or similar populations during the same time period $(n=49,92 \%)$, and had a timeframe that was sufficient to reasonably expect to see an association between exposure and outcome $(n=43,81 \%)$ (Table 2). However, 44 studies did not provide a sample size justification, power description or variance and effect estimate $(83 \%)$, and 17 studies did not measure or adjust for confounding variables for their impact on the relationship between exposure and outcomes (32\%). Thirty-five studies did not measure the exposure variable more than once over time ( $n=35 / 40,88 \%)$.

\subsection{Work-Related Outcomes}

Out of all included papers $(n=53)$, outcomes related to RTW were reported by 27 studies (51\%), sick leave by 23 studies (43\%), work capacity by 13 studies $(25 \%)$, work ability by 6 studies (11\%) and productivity loss by 4 studies (8\%) (Supplementary Material S2). Pooled prevalence and quality of evidence are summarised according to outcomes of RTW, sick leave and work capacity. Meta-analysis was not possible for work ability and productivity loss.

\subsubsection{Return to Work}

RTW proportions were reported at different time points, ranging from 1 week to 3 years (Table 3). Six studies did not specify a specific RTW time point (Table 3) $[29,47,62,74,77,80]$. Pooled RTW percentages showed a RTW prevalence of $69 \%$ (95\% CI 47, 88, I $299 \%$; 4 studies) up to 1 month, $67 \%$ (95\% CI 45, 86, I $\mathrm{I}^{2} 91 \%$; 3 studies) up to 3 months, $76 \%$ (95\% CI 48, 95, $\mathrm{I}^{2}$ 99\%; 4 studies) up to 6 months, $83 \%$ (95\% CI 69, 94, I $\mathrm{I}^{2} 99 \%$; 10 studies) at 12 months and $70 \%$ (95\% CI 52, 85, I I 96\%; 5 studies) at 24 months (Supplementary Material S3; Figure 2). Pooled percentages at all time points had high statistical heterogeneity $(>91 \%)$ (Supplementary Material S3). 
Table 2. Quality assessment with the National Heart, Lung, and Blood Institute Study Quality Assessment Tools For observational cohort and cross-sectional studies.

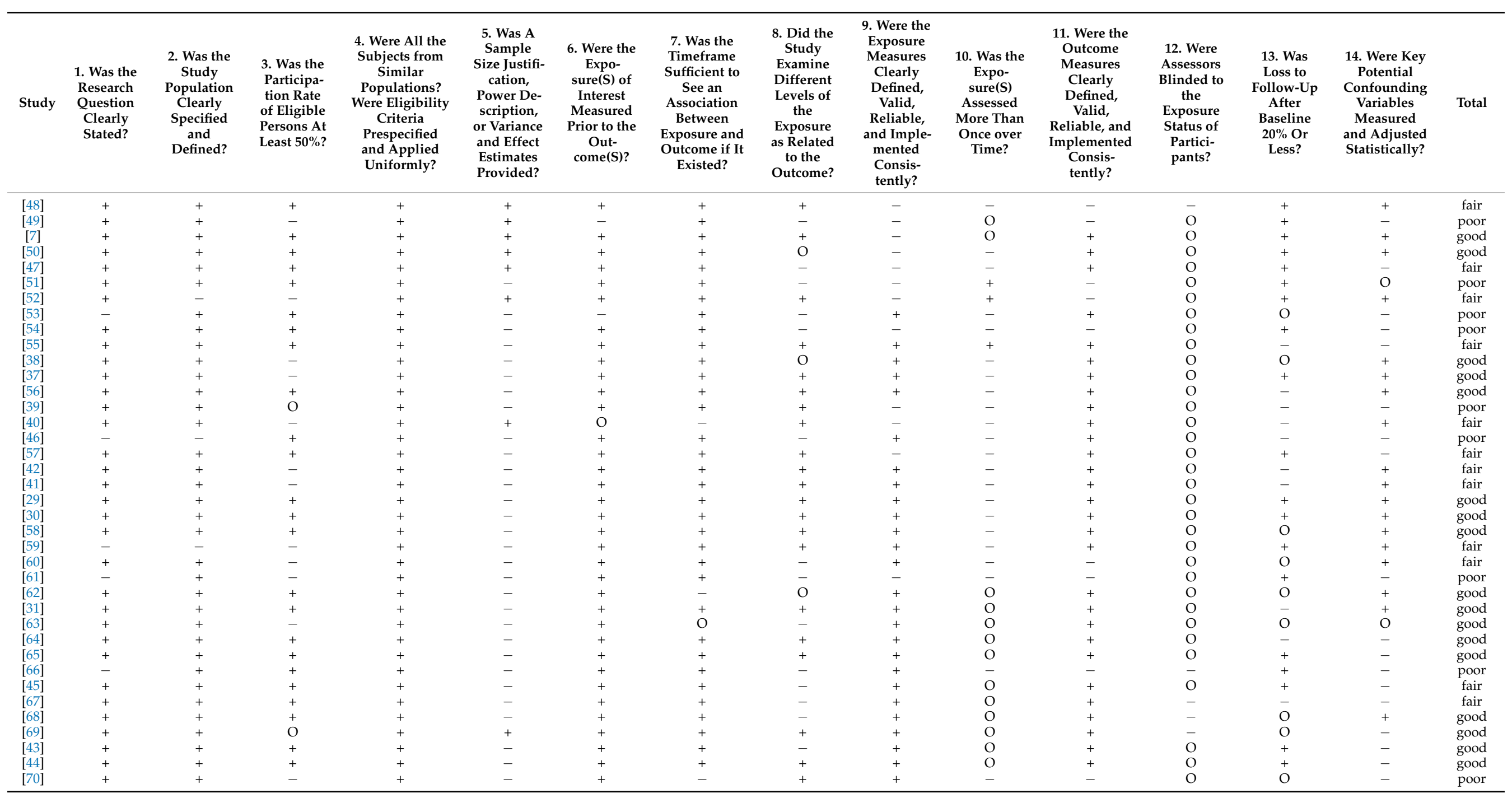


Table 2. Cont.

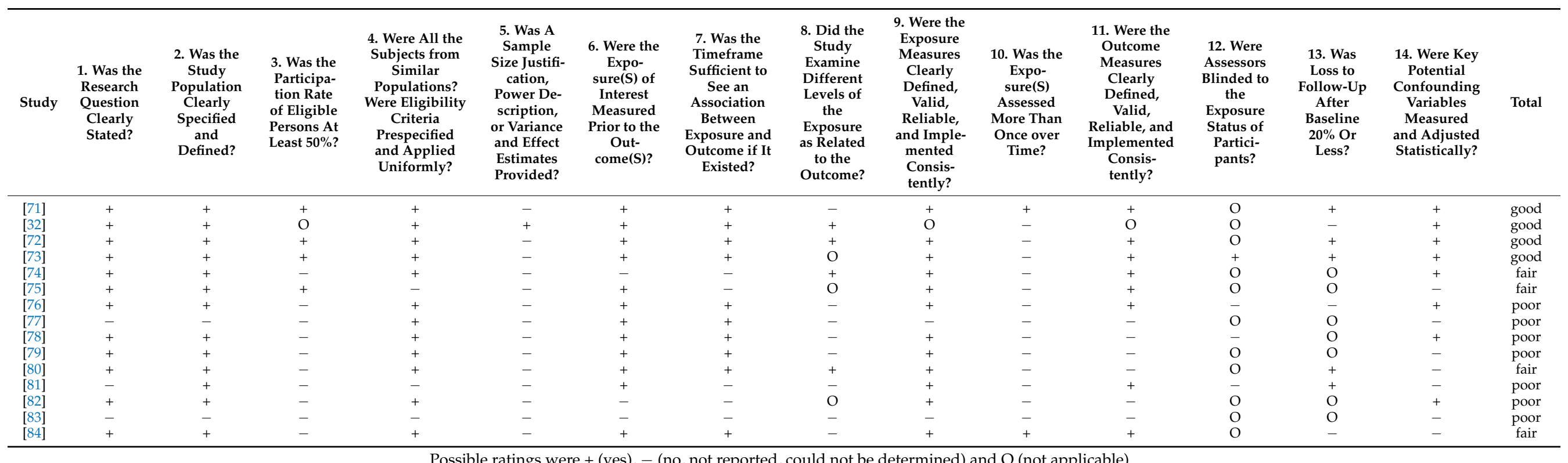


Table 3. Return to work percentages by time point.

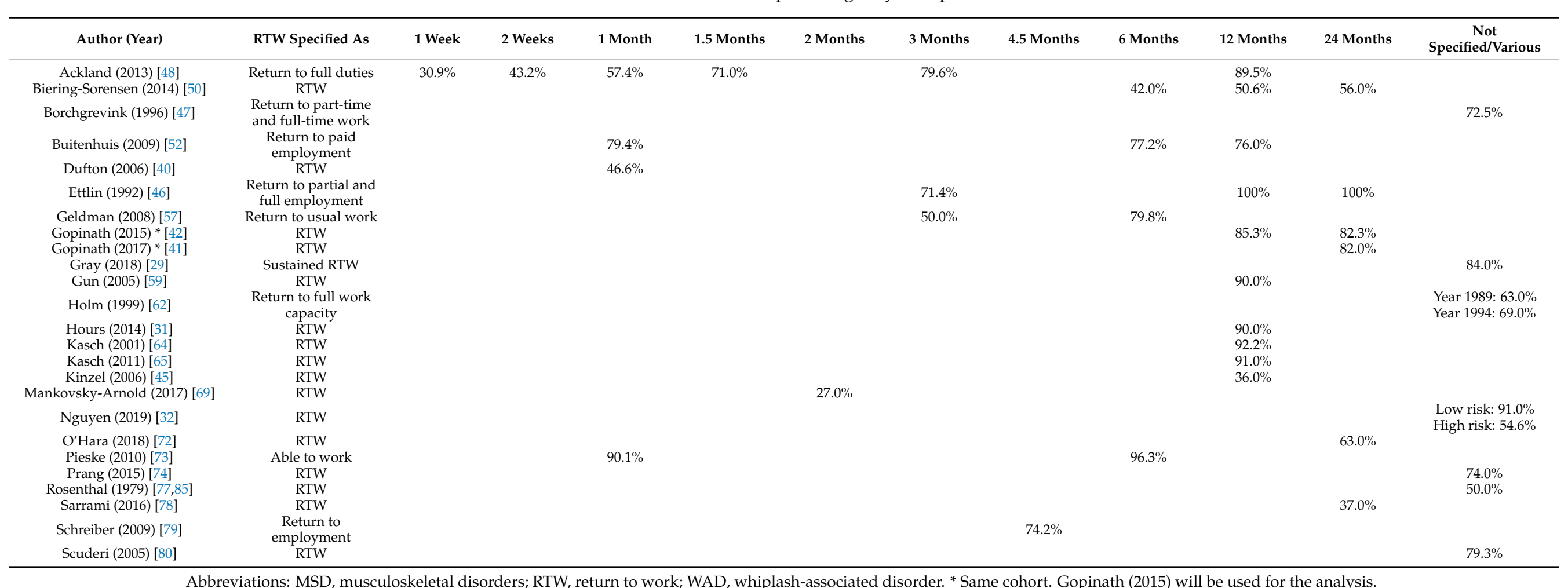

Abbreviations: MSD, musculoskeletal disorders; RTW, return to work; WAD, whiplash-associated disorder. * Same cohort. Gopinath (2015) will be used for the analysis. 


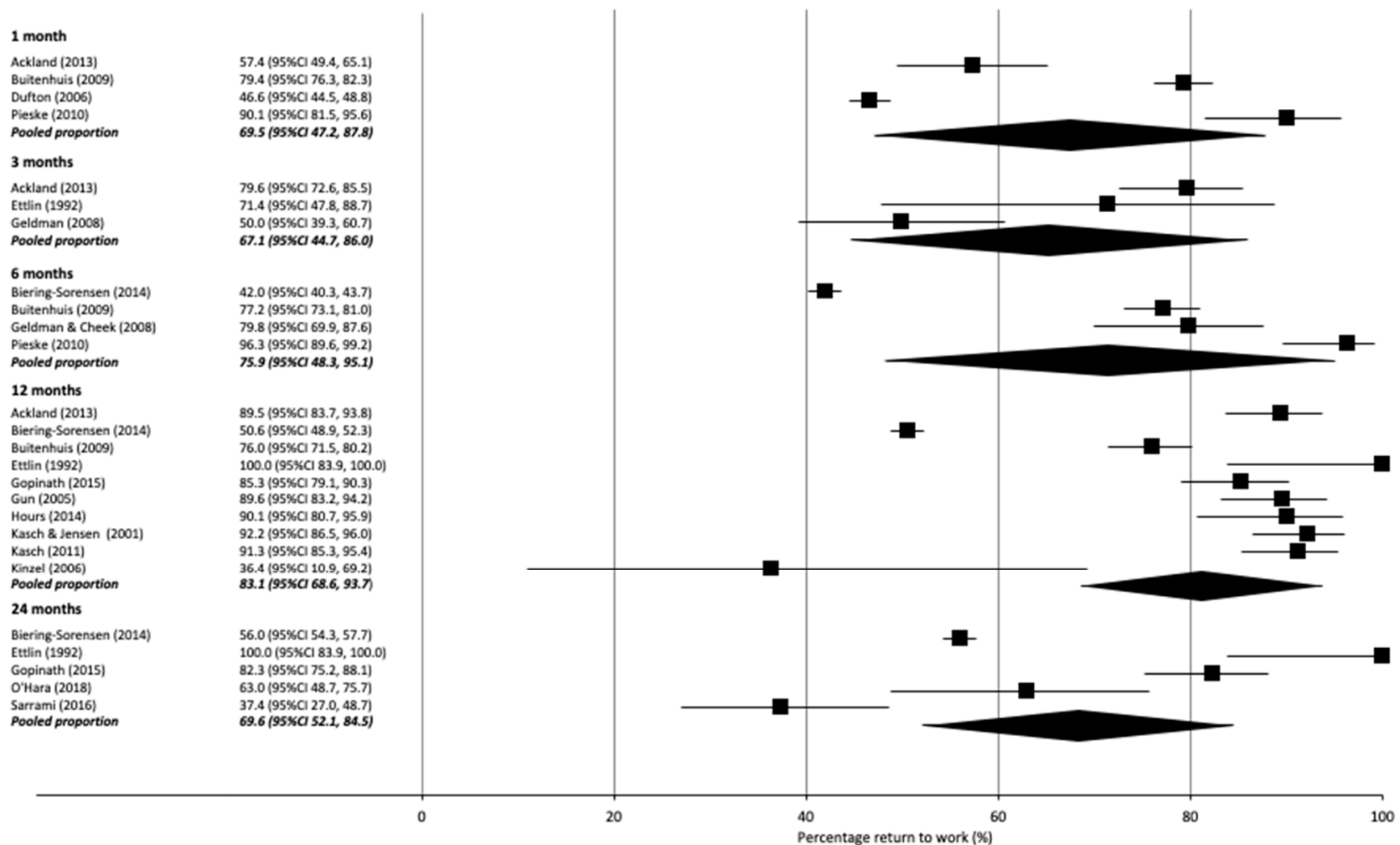

Figure 2. Forest plot with percentages and pooled percentage of RTW as reported per month.

\subsubsection{Sick Leave}

Sick leave was reported as percentages for a certain duration and as percentages reported at a certain time point (Table 4). Three studies did not report a specific time point or duration (Table 4) $[7,39,83]$.

Sick leave was reported at different time points, ranging from 1 month to 2 years (Table 4). Pooled percentages revealed that $27 \%$ (95\% CI 16, 41; I $97 \%$; 4 studies) of injured workers had used sick leave at 1 month post-RTC, $13 \%$ (95\% CI 6, 24; I $\mathrm{I}^{2} 94 \% ; 3$ studies) at 3 months, 23\% (95\% CI 0, 65; I $\mathrm{I}^{2} 100 \%$; 5 studies) at 6 months, $26 \%$ (95\% CI 20, 54; $\mathrm{I}^{2}$ 99\%; 5 studies) at 12 months and 22\% (95\% CI 13, 33; I $\mathrm{I}^{2} 94 \% ; 4$ studies) at 24 months (Supplementary Material S4; Figure 3).

Pooled sick leave of less than 2 weeks was reportedly used by $28 \%$ of injured workers (95\% CI 9 to 53; I $\mathrm{I}^{2}$ 98\%; 5 studies), between 2 and 4 weeks by $12 \%$ (95\% CI 8, 16; I $\mathrm{I}^{2} 41 \%$; 3 studies), and sick leave of more than 4 weeks was reported by $15 \%$ (95\% CI 11, 20; I $\mathrm{I}^{2} 46 \%$; 4 studies) (Supplementary Material S4; Figure 4). Injured workers in two studies reported a mean sick leave duration of 6.6 days (no estimate of variance reported) [51] and 13.5 days (SD 29.8) [56] at 6 months post-RTC. Another study reported a duration of 2-3 weeks of sick leave at 12 months [60], and a retrospective study reported a mean leave of absence of 104 days (range 24-382) [70]. Further information regarding how sick leave was measured and reported is available in Supplementary Material S2. 
Table 4. Sick leave percentages reported as duration of sick leave or used sick leave at a certain time point.

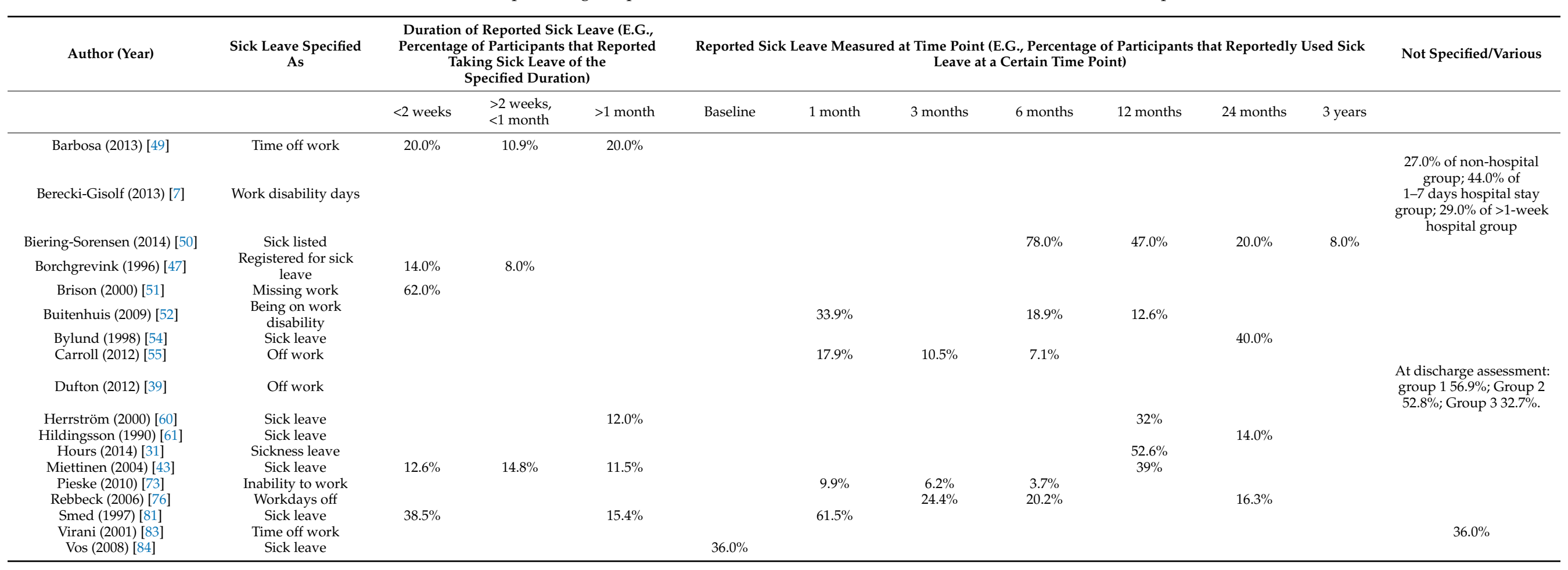




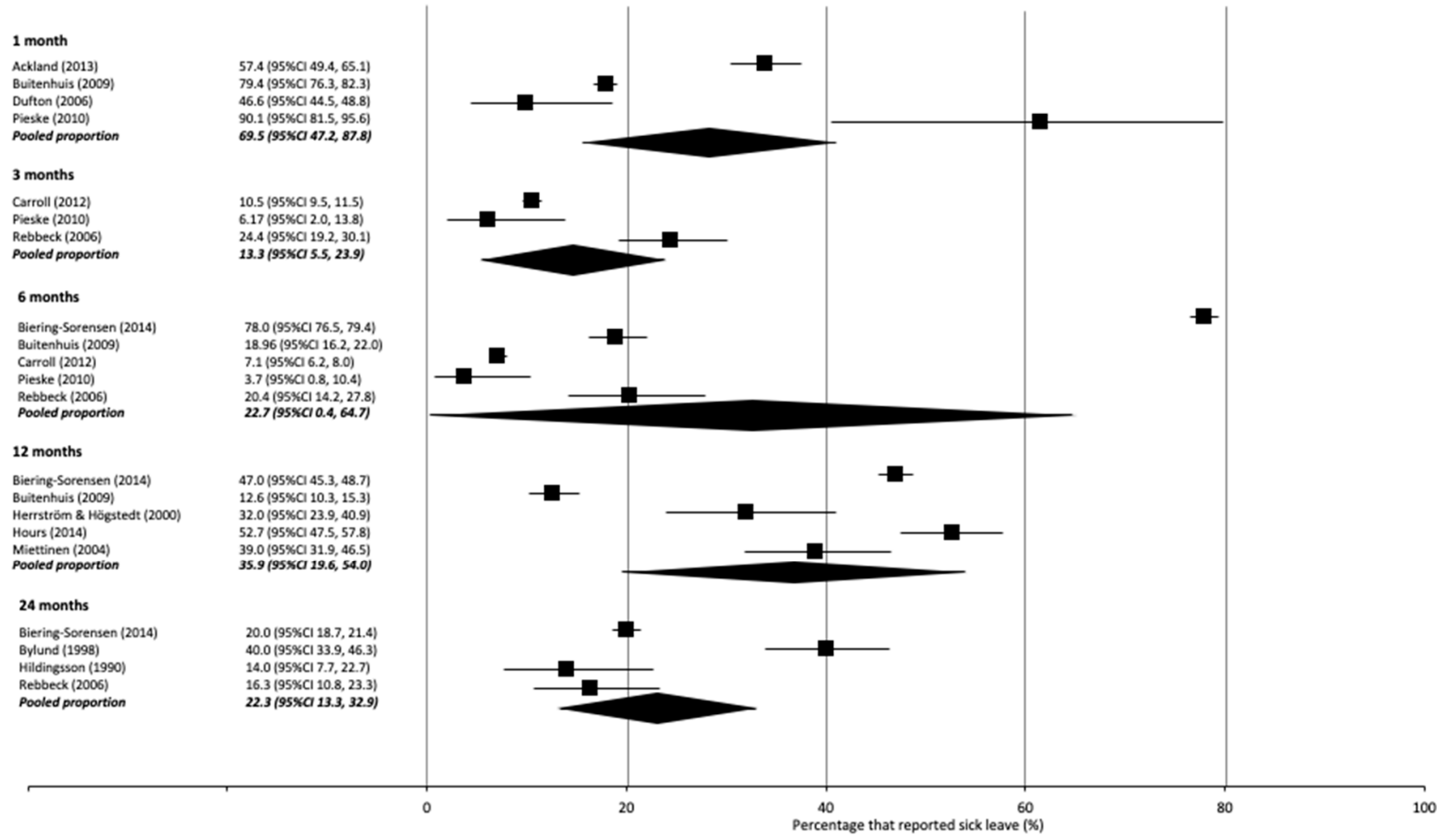

Figure 3. Forest plot with percentages and pooled percentage of participants taking sick leave as reported per time point $(1,3,6,12$ and 24 months).

\begin{tabular}{|c|c|}
\hline \multicolumn{2}{|l|}{$<2$ weeks of sick leave } \\
\hline Barbosa (2013) & 20.0 (95\% 14.2 \\
\hline Borchgrevink (1996) & 13.9 (95\%वl 8.2, 2 \\
\hline Brison \& Pickett (2000) & 62.0 (95\%Cl 56.8, \\
\hline Miettinen (2004) & $12.6(95 \% \subset$ ) $8.2,1$ \\
\hline Smed (1997) & $38.5(95 \% \subset 120.2$ \\
\hline Pooled proportion & 28.1 (95*a 8.9, s \\
\hline \multicolumn{2}{|c|}{$>2$ weeks, $<1$ month of sick leave } \\
\hline Barbosa (2013) & 10.9 (95\%व $6.6,1$ \\
\hline Borchgrevink (1996) & $7.8\left(95 \mathrm{SCl}_{3.6} .6 .14 .3\right)$ \\
\hline Miettinen (2004) & 14.8 (95\%) 10.0, \\
\hline Pooled proportion & $11.6(95 \% c 18.1,1$ \\
\hline \multicolumn{2}{|l|}{$>1$ month of sick leave } \\
\hline Barbosa (2013) & 20.0 (95\%Cl 14.2, \\
\hline Herrstròm \& Högsteđt (2000) & 12.0 (95\%:C1 6.9 \\
\hline Miettinen (2004) & $11.5(95 \% C 1) 7.3$ \\
\hline Smed (1997) & $15.39(95 \% \mathrm{Cl} 4.4$ \\
\hline Pooled propc & $14.8(95 \% a 10.6$ \\
\hline
\end{tabular}

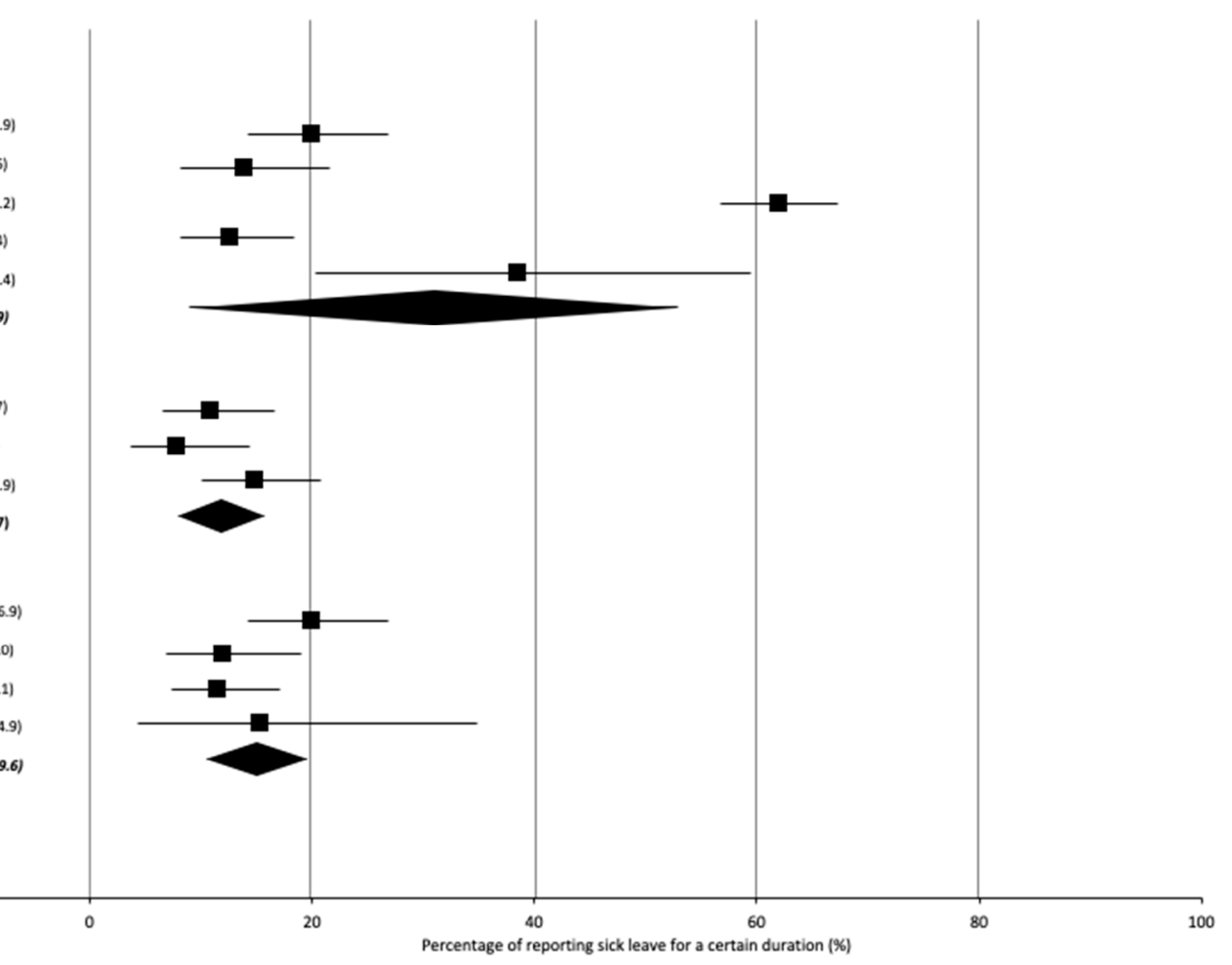

Figure 4. Forest plot with percentages and pooled percentage of participants reporting sick leave for a certain duration. 


\subsubsection{Work Capacity}

Eleven studies (thirteen publications) reported outcomes related to modifying duties at work and/or the inability to work [61] for time periods up to 6 months post-RTC [51] (Supplementary Material S2). One study reported that $38 \%$ of participants were unable to continue pre-injury work within 3 months of injury [38] and the pooled percentage from two other studies showed that $10 \%$ of participants did not return to pre-injury work capacity at 1 year post-RTC (pooled percentage 10\%, 95\% CI 1 to 20) [66,67] (Supplementary Material S2). Gopinath et al. (2015) reported that $82 \%$ of participants resumed full duties at 12 months and $89 \%$ at 24 months [42]. In contrast, in a study with a lower RTW rate (37\%) at 2 years, $23 \%$ had returned to pre-injury duties at 2 years [78]. Other studies report periods of modified duties [48], rates of modified duty use [51], and results by injury chronicity [39] (see Supplementary Material S2).

\subsubsection{Work Ability}

Six studies $[48,53,63,71,75,79]$ described outcome measures consistent with work ability. There was no consistency of measurement tools between the six studies, and they were rarely validated, as such a meta-analysis was not conducted. Instead findings are reported narratively.

Percentage of participants with reduced work ability, defined as 'capability' (based on a combination of sick leave and reduced work hours) was $15 \%$ at 12 months in one study [71]. When reduced work ability was defined as 'decreased work function' (selfreported limitation in ability to work) [79], the prevalence was 34\% at mean 4.5 months $(n=38)$ (Supplementary Material S2). Bunketorp and Carlsson used insurance physicians to assign a degree of medical disability resulting from crash-related neck injury to 18 of 25 participants who had residual neck pain and an insurance claim [53]. When functional capacity evaluations were combined with medical chart review in the definition of work ability, 26 of 76 patients (34\%) with WAD were rated as having work disability at mean 2.4 (SD 22) years (range $0.4-13$ years) [75].

Hoving and colleagues (2003) [63] reported results in a cross-sectional study for the work-specific items within the Neck Disability Index (mean (SD) 2.2 (1.3) where $0=$ no disability and $5=$ total disability) and Northwick Park Neck Pain Questionnaire (mean (SD) 1.7 (1.2) where $0=$ no difficulty and $4=$ severe difficulty) in people with WAD from 0 to $>24$ months post-injury. Patients with neck pain were asked to nominate their reasons for their delay in returning to full work duties in a study by Ackland and colleagues [48]. Of the $69 \%$ of patients who did experience a delay, $50 \%$ nominated their neck injury or neck pain as the reasons, whilst $12 \%$ nominated other crash-related injuries, and $7 \%$ nominated psychological issues.

\subsubsection{Productivity Loss}

Four studies reported measures of health-related productivity loss [49,58,68,72]. Absenteeism was reported by one study in $n=13 / 15$ participants with facial injuries [49]. Those who experienced psychological distress in conjunction with their musculoskeletal injury had statistically similar rates $(p=0.96)$ of not filing for an economic loss claim $(n=329$, $54.2 \%)$ compared with those who had musculoskeletal injury only $(n=3101,54.1 \%)$ [58]. At 2 years post-RTC, monthly income was $62 \%$ less than pre-injury monthly earnings (mean difference, USD $\$ 117.50 ; 95 \%$ CI USD $\$ 34$ to USD $\$ 201$ ) for those with tibial/femoral fractures in Uganda [72]. Of 1203 participants with whiplash in Denmark, 47\% experienced lost earnings capacity in the 5 years following RTC [68] (see Supplementary Material S2).

\subsection{Sensitivity Analysis of Work-Related Outcomes}

Return to work outcomes were robust to sensitivity analysis (i.e., excluding studies rated as 'poor') for 1, 3, 6, and 24 months but not for 12 months (all studies 83.05\%, moderate and good quality studies 80.62\%, $p<0.01$ ) (Supplementary Material S5). Sick leave duration was robust to sensitivity analysis, except for sick leave duration $<2$ weeks 
(all studies $28.10 \%$, moderate and good quality studies $13.37 \%, p<0.001$ ) (Supplementary Material S6). Sick leave percentages reported at 6 and 12 months were robust to sensitivity analysis, but not 1 month $(27.32 \%$ vs. $20.40 \%, p<0.001)$ and 3 months $(13.32 \%$ vs. $9.76 \%$, $p<0.001$ ) (Supplementary Material S6). Three out of four studies at 24 months were rated as poor quality, therefore sensitivity analysis was not possible.

\subsection{Factors Associated with Work-Related Outcomes}

Fifty-six percent of all studies ( $n=30 / 53$ ) reported information on (potential) factors associated with work-related outcomes (Supplementary Material S7). Of the studies that reported RTW, 52\% $(n=14 / 27)$ investigated associated factors. For sick leave, 39\% $(n=9 / 23)$ investigated associated factors, for work capacity $23 \%(n=3 / 13)$, for work ability $50 \%(n=3 / 6)$, and for productivity loss $75 \%(n=3 / 4)$ of studies investigated associated factors (Supplementary Material S7).

\subsubsection{Return to Work}

Risk factors for delayed return to work were predominantly health-related including injury type (whiplash, dislocations vs. limb fracture), greater severity of injury, being admitted to hospital, having a higher baseline OMPSQ score, having lower baseline mental health-related quality of life, lower pre-injury fitness levels and the presence of a pre-injury chronic illness $[29,32,42,57]$. Sociodemographic characteristics such as lower socioeconomic status, lower education and being a widow, separated or divorced were also risk factors for delayed return to work [30,74]; however, the findings for age and sex on return to work were mixed $[30,42,74]$. Support from employers was positively associated with returning to work [74]. Consulting a lawyer was associated with a 5-fold lesser chance of returning to work at 1 year, but this association was not significant after adjusting for the neck pain outcome score, bodily pain scores and role emotional scores [59].

\subsubsection{Sick Leave}

Two studies reported associations between taking time off work/sick leave and being female $[7,54]$ and two studies reported that women took longer average sick leave than men $[44,54]$. Longer sick leave was reported in participants who were married, divorced or widowed compared to single persons $(p<0.05)$ [43]. No significant associations on the length of sick leave were found in Miettinen et al. [43] with age, education, speed of the vehicle, position in the car or use of the seatbelt. Receiving workers compensation for neck pain after RTC was associated with more days off work compared with seeking compensation via a personal injury claim [80].

Four studies investigated the relationship between injury-related factors and sick leave. Sustaining a whiplash injury was a risk factor for taking more sick leave [54] and not recovering from a whiplash injury was a risk factor for being on sick leave [53], although one study found no difference in length of sick leave between those without whiplash, with grade 1 or with grade 2 whiplash [31]. Level of protective equipment worn by motorcycle riders (unprotected, partial, or full protection) was not related to number of days off work [56].

\subsubsection{Work Capacity}

One study reported that those who were unable to continue in their pre-injury work capacity scored significantly worse on all health outcome measures including the SF-36 and pain catastrophizing scale [37], whereas another study reported that there were no significant associations between work capacity and potential predictors including age, gender, surgery type and location, and their socioeconomic indexes area [78]. Holm et al. [62] reported that all participants with partial or full work disability were over 40 years of age, had over $15 \%$ of medical impairment and a lower professional status than those with no work disability. 


\subsubsection{Work Ability}

One study reported no evidence of an association between gender and status as work disabled $(p=0.12)$ [53]). As reported by Myrtveit et al. [71], reduced work capacity was associated with preferring to take medications, sickness absence, being referred to a physiotherapist or chiropractor; whereas participants who believed the active coping preferences of "living as usual" and "change of lifestyle" could make them better were protective for reduced work capacity.

\subsubsection{Productivity Loss}

Barbosa et al. [49] reported a significant association between the occurrence of a facial injury and absenteeism $(p=0.024)$. An economic analysis of data from Denmark found those with WAD injuries who were awarded compensation on the basis of injury severity were more likely to have lost earning capacity in the long term [68]. O'Hara et al. [72] reported no association between surgical treatments, monthly income, debt, employment or dependents (Supplementary material S7).

\subsection{Impact of Deviation from Protocol on Results}

As described in the methods Section 2.2.1, four studies that included adolescents in their cohorts were included in this review as a deviation from the study protocol [29-32]. The four studies that were included with adolescent participants did not limit the collection of RTW data within their study to adults ( $>18 \mathrm{y}$ ) only, and results were not presented separately for adolescents and adults, meaning data could not be extracted for adults only. If the adolescents were not previously working prior to their RTC, they were not included as having RTW in these studies; and if they were employed prior to their RTC, their postinjury RTW status is also of interest. These studies treated any adolescents in their cohorts as adults; this is evident in the manner in which the cohort characteristics were described. The presentation of the cohort characteristics does not clearly state what percentage of the cohort was represented by adolescents; however, it is likely to be small. This does restrict us from making any comparison across key confounders or outcomes between adolescents and the adults in the wider review. Two studies by Gray and colleagues [29,30], based on the same dataset, reported $22 \%$ of their cohort was aged 15-24 y, and mean age is not given. The study by Hours and colleagues [31] reported $21 \%$ of their cohort was aged 16-24 y, and mean age is not given. The study by Nguyen and colleagues [32] included $n=498$ total from the age of $17 \mathrm{y}$ and above, with a mean (SD) age of 40.2 (16) y for neck injuries, 35.7 (17) y for lower back injuries, and 38.9 (15) for lower limb injuries. Any $17 \mathrm{y}$ old participants would have been more than 1 standard deviation outside of the mean. In a normally distributed dataset, $68 \%$ of the cohort is within one standard deviation of the mean (mean $+/-1 \mathrm{SD})$.

The potential impact of including these four studies on the pooled meta-analyses is minimal. Only one study [32] contributed data to meta-analyses (see Figures 2 and 3). Results from this study by Nguyen and colleagues [32] for the rate of RTW at 12 months was consistent with several other studies in the meta-analysis $[59,65,66]$. The use of sick leave at 12 months was highest in this study across the five studies in this meta-analysis, however likely balanced out in the meta-analysis by a corresponding low usage rate from another study [52].

\section{Discussion}

This systematic review identified 53 observational studies in which RTW, sick leave, work capacity, work ability, and health-related work productivity loss were measured in adults with a musculoskeletal injury after RTCs. Findings from the meta-analyses within this review should be interpreted with caution, as the degree of statistical heterogeneity was high. Each pooled statistic was the product of the combination of different studies, and represents a combined estimate based on similar data drawn from at least two studies. Outputs from those meta-analyses demonstrated the pooled RTW rate generally increases 
post-injury from $70 \%$ of injured persons having RTW at 1 month, to $67 \%$ at 3 months, $76 \%$ at 6 months, and $83 \%$ at 12 months. These findings suggest that most injured persons are back at work within a month of their RTC, and of those who are not, some go on to return gradually to work over the first year after RTC, whilst some will not have a successful RTW at 1 year post-injury. Approximately one-third of injured workers used sick leave in the year after RTC, and approximately one-sixth used more than 4 weeks of sick leave. Using sick leave may be the best (or only) option for individuals without suitable modified duties available to them in their workplace. Results of four studies demonstrated a varying percentage (though always $<50 \%$ ) of workers used modified duties at work. Few studies identified by this review measured work ability ( 6 of 50 studies, $12 \%$ ) or health-related work productivity loss (4 of 50 studies, $8 \%$ ). More research is needed in this client population regarding these outcome measures for appropriate conclusions to be drawn.

There are several key aspects of the methodology of the included studies that are worth highlighting, and should be considered with the findings. It is encouraging to have a high percentage of prospective observational studies within this review, and many with large sample sizes from administrative databases, which can both be considered as markers of methodological quality within the field. On the other hand, administrative databases may be biased representations of the total population of adults with musculoskeletal injuries after RTC, over-emphasising the experience of those who presented to hospital (ED/hospital datasets) or those who were eligible for compensation (insurance databases), depending on the jurisdiction in which they live. Regarding type of injury, the most common injury type were whiplash injuries. This fits with the frequency of whiplash injuries in RTC and the emphasis placed on these injuries in the literature [1,85-87]. It must be acknowledged that as a result the work-related findings of this review are most relevant to those with whiplash injuries and are less generalizable to musculoskeletal injuries as a whole. Developed countries such as Canada, Australia and Denmark may also be overrepresented in the results-only three studies were from the developing nations [88] of Uganda [72], Brazil [49], and Chile [70]. A report published by the Global Road Safety Facility in 2014 described an annual total of 78.2 million non-fatal injuries in less developed regions such as Sub-Saharan Africa [89], therefore future research on the employment impacts of RTC are needed from these regions to better represent the global burden of traffic injuries. Many studies did not justify their sample size, either with a power calculation or an indication of what percentage of eligible individuals consented to participate. Absence of a priori sample size calculations in observational studies has implications for detecting a difference in the primary outcome between sub-groups. In addition, many studies did not provide an indication of how representative their sample was of the true population of those injured by RTCs by omitting a comparison between those who consented versus those who declined to participate. However, the use of data such as the age and sex of those who declined to participate may not have been available to researchers, as there are ethical concerns related to the collection of data from those who have not consented.

Of the studies reporting RTW rates, the most data were available for 12 months postinjury, demonstrating that $83 \%$ of adults with a musculoskeletal injury after RTC had returned to work by 12 months. RTW rates range from 1 month (69.5\%) to 12 months (83\%) and suggests that those who have not returned by 1 month may take several months to achieve a successful RTW. Vocational rehabilitation programs can facilitate RTW by optimising work participation usually with a combination of medical, psychological, social and/or occupational strategies: for example, assessments of functional capacity and vocational goals, vocational counselling, training and work experience opportunities (particularly within the workplace), and assistance with job seeking [90]. Findings from this review suggest that there is a need for vocational rehabilitation services across the first year after RTC. Existing evidence from the field of occupational injuries cannot be assumed to apply to those injured in RTCs, as the legislated requirements for support from an employer after a work-related injury may differ to those following RTC-related injury. 
Existing evidence for vocational rehabilitation, and rehabilitation more broadly, also needs to expand to encompass developing nations. The World Health Organization's 'Decade of Action for Road Safety 2021-2030' calls for the provision of rehabilitation to all RTC injured persons, and particularly for "protections for people with disabilities to keep their jobs or be hired in new jobs through the provision of incentives for employers will further alleviate the socioeconomic consequences of permanent disability"[91]. Liability insurance could contribute greatly to this vision; for example in Uganda in 2020, a third party insurance compensation scheme was established to help victims of RTCs pay for medical expenses [92]. Road traffic injuries place a significant burden on the health care systems of developing nations, where additional socioeconomic consequences flow on from loss of employment and permanent disability [93].

Interestingly, the pooled data (5 studies) for 24 months post-injury resulted in the same RTW rate as one month post-injury-70\%. This could reflect a portion of injured persons being unable to sustain their RTW, which is known to occur [29]. This could also be due to methodological issues with drop out such that individuals who have not yet RTW at 2 years may be more likely to have time to engage in research participation than those who have RTW. The needs of these individuals attempting to remain at work during their recovery are not well understood, and are worthy of further exploration.

Inconsistency in the definition of RTW, with no universally accepted standard, makes it difficult to carry conclusions across different types and mechanisms of injuries. In this review, some studies considered return to exactly the same job, hours and/or conditions (e.g., [48,57,62]), while other studies defined RTW as return to any form of paid employment, including different roles, reduced hours and modified duties (e.g., [42,46]). More detail and consistency in the definition of RTW are needed in future research studies. This definition may also reflect the method of data collection. Studies may use self-reported data regarding RTW, and therefore RTW becomes open to interpretation by the participant themselves if clear instructions are not provided by the investigators. While administrative databases are likely to provide more consistency in how RTW is defined, those definitions are still likely to differ between countries (e.g., Australia [29] vs. Denmark [68]).

Meta-analyses of sick leave data demonstrated approximately 1 in 4 injured persons used sick leave in the first year after their RTC, and for those who did use sick leave, 15\% used it for more than 4 weeks. Musculoskeletal disorders in general are responsible for a significant amount of sickness absence and work disability, with the associated loss in productivity being equivalent to an estimated 2\% of the European Union's gross domestic product [94]. In a Swedish study of individuals who suffered severe physical trauma, 11\% of individuals were on full-time sick leave 12 months after their injury [95]. In this review, the RTW rate at 1 month post-injury was 70\%, and the proportion of injured persons using $>4$ weeks of sick leave was $15 \%$. This may indicate a gap for $~ 15 \%$ of injured persons who are unable to work, and who may or may not be able to source other forms of income, such as government supportive payments or insurance payouts. Financial strain after RTC is a significant concern: some may find ongoing medical costs a cause of bankruptcy [96]. To avoid bankruptcy, some workers make the decision to RTW before their illness or injury has recovered [97]. Casualization of workforces [98] may be another potential reason behind the early RTW of those who are still ill/injured, and the $\sim 15 \%$ of persons unable to RTW or to access paid sick leave. The jurisdiction in which an injured person resides is an important factor in determining access to financial support. For example, in Australia, the state of Victoria has a no-fault based scheme that pays benefits to injured persons in the form of wage replacement when they are unable to work [99]. In contrast, the state of Queensland has a fault-based scheme that does not pay wage replacements during the life of a claim, rather including income lost in the final claim amount [100]. In the present review, one study identified individuals with neck pain after RTW took more days off if they were receiving workers compensation in comparison to compensation via a personal injury claim [80]. More research into the relationship between fault status, compensation status, and work outcomes is needed. 
A wide range of domains were assessed and found to be associated with the workrelated outcomes within this review. Categories of independent variables included sociodemographic factors, psychosocial health, injury and RTC characteristics, pre-crash mental and physical health, educational attainment, employer support, and type of work. The significance of any association, and if present, the direction of the association varied for many of these characteristics across multiple work-related outcomes. There are some links that could be drawn from the results of the present review; for example, people who were married, divorced or widowed were more likely to have longer sick leave (vs single people) [43] and a delayed RTW (vs never married) [74]. Gender (or sex) was highly variable (and sometimes used interchangeably), being associated with taking sick leave [7,43,54], having no evidence for an association with work ability or work capacity [53,78], and having mixed results for an association with RTW $[29,42]$. The systematic review by Samborec and colleagues [101] examined the relationship between biopsychosocial factors and non-recovery following minor RTC-related injury. In this review, RTW was one of the many outcomes that could be considered as a measure of recovery. Similarly to the present review, there was conflicting evidence for the association of sex (female/male) with non-recovery [101]. The strongest evidence for an association with non-recovery was found for characteristics of pain (initial intensity, duration, severity) and pre-injury mental and physical health [101]. Results of both of these reviews highlight the multifactorial nature of recovery and RTW-recently supported by the outcomes of our Delphi study [102], and the difficulty in predicting when an individual will RTW. Support to RTW or to recover more broadly after RTC should therefore be individualised.

This review has a number of strengths. A thorough database search was conducted across five scholarly databases and one grey literature source specific to the transport industry. Independent review by two co-authors for screening, data extraction and appraisal led to high ratings of agreement. Results were presented by timeframe, to aid in the interpretation of recovery after RTC over time. Finally, five work-related outcomes were investigated, expanding the knowledge base beyond a focus on achievement of RTW.

There are also limitations that must be acknowledged. There were high levels of heterogeneity in the results of the meta-analyses. The database search was restricted to studies published in English which may have contributed to the under-representation of results from developing countries. There are a number of validated tools for measuring work capacity and work ability that this review was expecting to find [26]. However, few studies measured either of these concepts comprehensively and when they did, it was often to report a rate of work incapacity or inability defined to suit the individual study and lacking evidence of the psychometric properties of the outcome. Many of the studies included in this review recruited only patients with whiplash injuries post-RTC, making these results more applicable to injured persons with this particular musculoskeletal condition. On the whole, the cohorts included in this review could be said to represent mild to moderate musculoskeletal injuries, and not musculoskeletal injuries in general.

\section{Conclusions}

This review found that the pooled RTW rate increases in the first-year post-injury, from $70 \%$ at 1 month to $83 \%$ at 12 months. Approximately one-third of injured workers use sick leave in the year after RTC, and approximately one-sixth used more than 4 weeks of sick leave, reflecting a significant interruption to their employment and cost to their employer. Less than half of injured persons used modified duties at work following RTC-related musculoskeletal injury. More research is needed to understand the impact of RTC on work ability and health-related productivity loss. Clinicians are encouraged to consider the multiple potential factors of influence (health, sociodemographic, workrelated) for a client's recovery and RTW after RTC, and be familiar with the legal and insurance frameworks operating in their jurisdictions. 
Supplementary Materials: The following are available online at https: / www.mdpi.com/article/ 10.3390/ijerph182111504/s1, SM1: Search strategy; SM2: Work outcomes of RTW, sick leave, work capacity, work ability and productivity loss; SM3: Pooled RTW percentages by time point and quality of evidence; SM4: Pooled sick leave percentages, reported as duration of sick leave or used sick leave at a certain time point; SM5: Sensitivity analysis RTW proportions; SM6: Sensitivity analysis sick leave proportions; SM7: Factors associated with work-related outcomes.

Author Contributions: Conceptualization, E.M.G., C.L.B., E.J.S. and V.J.; methodology, E.M.G. and M.L.P.; formal analysis, C.L.B. and M.L.P.; resources, V.J.; data curation, E.M.G., M.L.P., C.L.B., E.J.S., T.A. and V.J.; writing — original draft preparation, E.M.G. and M.L.P.; writing—review and editing, C.L.B., E.J.S., T.A. and V.J.; visualization, M.L.P. All authors have read and agreed to the published version of the manuscript.

Funding: The RECOVER Injury Research Centre is a jointly funded initiative between the Motor Accident Insurance Commission (MAIC) and The University of Queensland (UQ). MAIC and UQ did not have any influence on the conduct, data analysis and interpretation, and dissemination of the final review report.

Institutional Review Board Statement: Not applicable.

Informed Consent Statement: Not applicable.

Data Availability Statement: Data sharing not applicable.

Conflicts of Interest: The authors declare no conflict of interest.

\section{References}

1. Holm, L.W.; Carroll, L.J.; Cassidy, J.D.; Hogg-Johnson, S.; Côté, P.; Guzman, J.; Peloso, P.; Nordin, M.; Hurwitz, E.; van der Velde, G.; et al. The burden and determinants of neck pain in whiplash-associated disorders after traffic collisions: Results of the Bone and Joint Decade 2000-2010 Task Force on Neck Pain and Its Associated Disorders. J. Manip. Physiol. Ther. 2009, 32, S61-S69. [CrossRef]

2. Cassidy, J.D.; Carroll, L.J.; Côté, P.; Lemstra, M.; Berglund, A.; Nygren, A. Effect of eliminating compensation for pain and suffering on the outcome of insurance claims for whiplash injury. N. Engl. J. Med. 2000, 342, 1179-1186. [CrossRef]

3. Connelly, L.B.; Supangan, R. The economic costs of road traffic crashes: Australia, states and territories. Accid. Anal. Prev. 2006, 38, 1087-1093. [CrossRef]

4. Motor Accident Insurance Commission. Statistical Information 1 July to 31 December 2016; Queensland Government: Queensland, Australia, 2016.

5. Gopinath, B.; Harris, I.; Nicholas, M.; Casey, P.; Blyth, F.; Maher, C.; Cameron, I. A Comparison of Health Outcomes in Older versus Younger Adults following a Road Traffic Crash Injury: A Cohort Study. PLoS ONE 2015, 10, e0122732. [CrossRef]

6. Jagnoor, J.; Wolf, A.; Nicholas, M.; Maher, C.; Casey, P.; Blyth, F.; Harris, I.; Cameron, I. Restriction in functioning and quality of life is common in people 2 months after compensable motor vehicle crashes: Prospective cohort study. Inj. Epidemiol. 2015, 2, 1-11. [CrossRef]

7. Berecki-Gisolf, J.; Collie, A.; McClure, R. Work disability after road traffic injury in a mixed population with and without hospitalisation. Accid. Anal. Prev. 2013, 51, 129-134. [CrossRef]

8. Norström, F.; Virtanen, P.; Hammarström, A.; Gustafsson, P.E.; Janlert, U. How does unemployment affect self-assessed health? A systematic review focusing on subgroup effects. BMC Public Health 2014, 14, 1310. [CrossRef]

9. Australasian Faculty of Occupational and Environmental Medicine. Realising the Health Benefits of Work: A Position Statement; The Royal Australasian College of Physicians: Sydney, Australia, 2011.

10. Waddell, G.; Burton, A.K. Is Work Good for your Health and Well-Being? Department for Work and Pensions: London, UK, 2006.

11. Mathers, C.D.; Schofield, D.J. The health consequences of unemployment: The evidence. Med J. Aust. 1998, 168, 178-182. [CrossRef]

12. Beales, D.; Fried, K.; Nicholas, M.; Blyth, F.; Finniss, D.; Moseley, G.L. Management of musculoskeletal pain in a compensable environment: Implementation of helpful and unhelpful Models of Care in supporting recovery and return to work. Best Pract. Res. Clin. Rheumatol. 2016, 30, 445-467. [CrossRef]

13. Vos, T.; Flaxman, A.D.; Naghavi, M.; Lozano, R.; Michaud, C.; Ezzati, M.; Shibuya, K.; Salomon, J.A.; Abdalla, S.; Aboyans, V.; et al. Years lived with disability (YLDs) for 1160 sequelae of 289 diseases and injuries 1990-2010: A systematic analysis for the Global Burden of Disease Study 2010. Lancet 2012, 380, 2163-2196. [CrossRef]

14. Dunstan, D.A.; Covic, T. Compensable work disability management: A literature review of biopsychosocial perspectives. Aust. Occup. Ther. J. 2006, 53, 67-77. [CrossRef]

15. WorkSafe Victoria. Clinical Framework For the Delivery of Health Services; Victorian WorkCover Authority: Melbourne, Australia, 2012. 
16. Hara, K.W.; Bjørngaard, J.H.; Jacobsen, H.B.; Borchgrevink, P.C.; Johnsen, R.; Stiles, T.C.; Brage, S.; Woodhouse, A. Biopsychosocial predictors and trajectories of work participation after transdiagnostic occupational rehabilitation of participants with mental and somatic disorders: A cohort study. BMC Public Health 2018, 18, 1014. [CrossRef] [PubMed]

17. Heron-Delaney, M.; Warren, J.; Kenardy, J.A. Predictors of non-return to work 2 years post-injury in road traffic crash survivors: Results from the UQ SuPPORT study. Injury 2017, 48, 1120-1128. [CrossRef]

18. O'Hara, N.N.; Isaac, M.; Slobogean, G.P.; Klazinga, N.S. The socioeconomic impact of orthopaedic trauma: A systematic review and meta-analysis. PLoS ONE 2020, 15, e0227907. [CrossRef] [PubMed]

19. Etuknwa, A.; Daniels, K.; Eib, C. Sustainable Return to Work: A Systematic Review Focusing on Personal and Social Factors. J. Occup. Rehabil. 2019, 29, 679-700. [CrossRef] [PubMed]

20. Papagoras, H.; Pizzari, T.; Coburn, P.; Sleigh, K.; Briggs, A.M. Supporting return to work through appropriate certification: A systematic approach for Australian primary care. Aust. Health Rev. Publ. Aust. Hosp. Assoc. 2018, 42, 164-167. [CrossRef]

21. El Fassi, M.; Bocquet, V.; Majery, N.; Lair, M.L.; Couffignal, S.; Mairiaux, P. Work ability assessment in a worker population: Comparison and determinants of Work Ability Index and Work Ability score. BMC Public Health 2013, 13, 305. [CrossRef]

22. Cameron, K.L.; Owens, B.D. The Burden and Management of Sports-Related Musculoskeletal Injuries and Conditions Within the US Military. Clin. Sport Med. 2014, 33, 573-589. [CrossRef]

23. Martimo, K.P.; Varonen, H.; Husman, K.; Viikari-Juntura, E. Factors associated with self-assessed work ability. Occup. Med. 2007, 57, 380-382. [CrossRef]

24. Campbell, P.; Wynne-Jones, G.; Muller, S.; Dunn, K. The influence of employment social support for risk and prognosis in nonspecific back pain: A systematic review and critical synthesis. Int. Arch. Occ. Env. Hea. 2013, 86, 119-137. [CrossRef] [PubMed]

25. Moher, D.; Liberati, A.; Tetzlaff, J.; Altman, D.G. Preferred Reporting Items for Systematic Reviews and Meta-Analyses: The PRISMA Statement. PloS Med. 2009, 6, e1000097. [CrossRef] [PubMed]

26. Gane, E.M.; Brakenridge, C.L.; Smits, E.J.; Johnston, V. The impact of musculoskeletal injuries sustained in road traffic crashes on work-related outcomes: A protocol for a systematic review. Syst. Rev. 2018, 7, 202. [CrossRef]

27. Gormley, M.; Devanaboyina, M.; Andelic, N.; Røe, C.; Seel, R.T.; Lu, J. Long-term employment outcomes following moderate to severe traumatic brain injury: A systematic review and meta-analysis. Brain Inj. 2019, 33, 1567-1580. [CrossRef] [PubMed]

28. Trenaman, L.; Miller, W.C.; Querée, M.; Escorpizo, R. Modifiable and non-modifiable factors associated with employment outcomes following spinal cord injury: A systematic review. J. Spinal Cord Med. 2015, 38, 422-431. [CrossRef]

29. Gray, S.E.; Hassani-Mahmooei, B.; Cameron, I.D.; Kendall, E.; Kenardy, J.; Collie, A. Patterns and Predictors of Failed and Sustained Return-to-Work in Transport Injury Insurance Claimants. J. Occup. Rehabil. 2018, 28, 740-748. [CrossRef] [PubMed]

30. Gray, S.E.; Hassani-Mahmooei, B.; Kendall, E.; Cameron, I.D.; Kenardy, J.; Collie, A. Factors associated with graduated return to work following injury in a road traffic crash. J. Transp. Health 2018, 10, 167-177. [CrossRef]

31. Hours, M.; Khati, I.; Charnay, P.; Chossegros, L.; Tardy, H.; Tournier, C.; Perrine, A.L.; Luaute, J.; Laumon, B. One year after mild injury: Comparison of health status and quality of life between patients with whiplash versus other injuries. J. Rheumatol. 2014, 41, 528-538. [CrossRef]

32. Nguyen, H.; Rebbeck, T.; Kifley, A.; Jagnoor, J.; Dinh, M.; Shetty, A.; Nicholas, M.; Cameron, I.D. Positive recovery for low-risk injuries screened by the short form-Orebro musculoskeletal pain screening questionnaire following road traffic injury: Evidence from an inception cohort study in New South Wales, Australia. BMC Musculoskelet. Disord. 2019, 20, 531. [CrossRef] [PubMed]

33. National Heart Lung and Blood Institute. Study Quality Assessment Tools. Available online: https://www.nhlbi.nih.gov/healthtopics / study-quality-assessment-tools (accessed on 20 May 2021).

34. Landis, J.R.; Koch, G.G. The measurement of observer agreement for categorical data. Biometrics 1977, 33, 159-174. [CrossRef]

35. Miller, J.J. The inverse of the Freeman-Tukey double arcsine transformation. Am. Stat. 1978, 32, 138.

36. Higgins, J.P.T.; Thompson, S.G.; Deeks, J.J.; Altman, D.G. Measuring inconsistency in meta-analyses. BMJ 2003, 327, 557-560. [CrossRef] [PubMed]

37. Casey, P.P.; Feyer, A.M.; Cameron, I.D. Associations with legal representation in a compensation setting 12 months after injury. Injury 2015, 46, 918-925. [CrossRef]

38. Casey, P.P.; Feyer, A.M.; Cameron, I.D. Identifying predictors of early non-recovery in a compensation setting: The Whiplash Outcome Study. Injury 2011, 42, 25-32. [CrossRef] [PubMed]

39. Dufton, J.A.; Bruni, S.G.; Kopec, J.A.; Cassidy, J.D.; Quon, J. Delayed recovery in patients with whiplash-associated disorders. Injury 2012, 43, 1141-1147. [CrossRef]

40. Dufton, J.A.; Kopec, J.A.; Wong, H.; Cassidy, J.D.; Quon, J.; Mcintosh, G.; Koehoorn, M. Prognostic factors associated with minimal improvement following acute whiplash-associated disorders. Spine 2006, 31, E759-E765. [CrossRef] [PubMed]

41. Gopinath, B.; Jagnoor, J.; Elbers, N.; Cameron, I.D. Overview of findings from a 2-year study of claimants who had sustained a mild or moderate injury in a road traffic crash: Prospective study. BMC Res. Notes 2017, 10, 76. [CrossRef]

42. Gopinath, B.; Jagnoor, J.; Harris, I.A.; Nicholas, M.; Casey, P.; Blyth, F.; Maher, C.G.; Cameron, I.D. Prognostic indicators of social outcomes in persons who sustained an injury in a road traffic crash. Injury 2015, 46, 909-917. [CrossRef]

43. Miettinen, T.; Airaksinen, O.; Lindgren, K.A.; Leino, E. Whiplash injuries in Finland-the possibility of some sociodemographic and psychosocial factors to predict the outcome after one year. Disabil. Rehabil. 2004, 26, 1367-1372. [CrossRef] [PubMed]

44. Miettinen, T.; Lindgren, K.A.; Airaksinen, O.; Leino, E. Whiplash injuries in Finland: A prospective 1-year follow-up study. Clin. Exp. Rheumatol. 2002, 20, 399-402. 
45. Kinzel, V.; Skirving, A.P.; Wren, M.N.; Zellweger, R. Sideswipe injuries to the elbow in Western Australia. MJA 2006, 184, 447-450. [CrossRef]

46. Ettlin, T.M.; Kischka, U.; Rechmann, S.; Radii, E.W.; Heim, S.; Wengen, D.; Benson, D.F. Cerebral symptoms after whiplash injury of the neck: A prospective clinical and neuropsychological study of whiplash injury. J. Neurol. Neurosur. PS 1992, 55, 943-948. [CrossRef]

47. Borchgrevink, G.E.; Lereim, I.; Royneland, L.; Bjorndal, A.; Haraldseth, O. National health insurance consumption and chronic symptoms following mild neck sprain injuries in car collisions. Scan J. Soc. Med. 1996, 24, 264-271. [CrossRef] [PubMed]

48. Ackland, H.M.; Cameron, P.A.; Wolfe, R.; Malham, G.M.; Varma, D.K.; Fitt, G.J.; Cooper, D.J.; Rosenfeld, J.V.; Liew, S.M. Outcomes at 12 months after early magnetic resonance imaging in acute trauma patients with persistent midline cervical tenderness and negative computed tomography. Spine 2013, 38, 1068-1081. [CrossRef]

49. Barbosa, K.G.; Lucas-Neto, A.; Gama, B.D.; Lima-Neto, J.C.; Lucas, R.S.; d'Avila, S. Injuries and absenteeism among motorcycle taxi drivers who are victims of traffic accidents. J. Forensic. Leg Med. 2014, 26, 15-18. [CrossRef]

50. Biering-Sorensen, S.; Moller, A.; Stoltenberg, C.D.; Holm, J.W.; Skov, P.G. The return-to-work process of individuals sick-listed because of whiplash-associated disorder: A three-year follow-up study in a Danish cohort of long-term sickness absentees. BMC Public Health 2014, 14, 113. [CrossRef]

51. Brison, R.J.; Hartling, L.; Pickett, W. A Prospective Study of Acceleration-Extension Injuries Following Rear-End Motor Vehicle Collisions. J. Musculoskelet Pain 2000, 8, 97-113. [CrossRef]

52. Buitenhuis, J.; de Jong, P.J.; Jaspers, J.P.; Groothoff, J.W. Work disability after whiplash: A prospective cohort study. Spine 2009, 34, 262-267. [CrossRef]

53. Bunketorp, L.; Nordholm, L.; Carlsson, J. A descriptive analysis of disorders in patients 17 years following motor vehicle accidents. Eur. Spine J. 2002, 11, 227-234. [CrossRef]

54. Bylund, P.; Bjornstig, U. Sick leave and disability pension among passenger car occpants injured in urban traffic. Spine 1998, 23, 1023-1028. [CrossRef] [PubMed]

55. Carroll, L.J.; Jones, D.C.; Ozegovic, D.; Cassidy, J.D. How well are you recovering? The association between a simple question about recovery and patient reports of pain intensity and pain disability in whiplash-associated disorders. Disabil. Rehabil. 2012, 34, 45-52. [CrossRef]

56. de Rome, L.; Ivers, R.; Fitzharris, M.; Haworth, N.; Heritier, S.; Richardson, D. Effectiveness of motorcycle protective clothing: Riders' health outcomes in the six months following a crash. Injury 2012, 43, 2035-2045. [CrossRef]

57. Geldman, M.; Moore, A.; Cheek, L. The effect of pre-injury physical fitness on the initial severity and recovery from whiplash injury, at six-month follow-up. Clin. Rehabil. 2008, 22, 364-376. [CrossRef]

58. Guest, R.; Tran, Y.; Gopinath, B.; Cameron, I.D.; Craig, A. Psychological distress following a motor vehicle crash: Evidence from a statewide retrospective study examining settlement times and costs of compensation claims. BMJ Open 2017, 7, e017515. [CrossRef]

59. Gun, R.T.; Osti, O.L.; O’Riordan, A.; Mpelasoka, F.; Eckerwall, C.G.E.; Smyth, J.F. Risk factors for prolonged disability after whiplash injury: A prospective study. Spine 2005, 30, 386-391. [CrossRef]

60. Herrström, P.; Lannerbro-Geijer, G.; Hogstedt, B. Whiplash injuries from car accidents in a Swedish middle-sized town during 1993-95. Scand J. Prim. Health Care 2000, 18, 154-158. [CrossRef]

61. Hildingsson, C.; Toolanen, G. Outcome after soft-tissue injury of the cervical spine. A retrospective study of 93 car-accident victims. Acta. Orthop. Scand 1990, 61, 357-359. [CrossRef]

62. Holm, L.; Cassidy, J.D.; Sjogren, Y.; Nygren, A. Impairment and work disability due to whiplash injury following traffic collisions: An analysis of insurance material. Scan J. Public Healt 1999, 27, 116-123. [CrossRef]

63. Hoving, J.L.; O'Leary, E.F.; Niere, K.R.; Green, S.; Buchbinder, R. Validity of the neck disability index, Northwick Park neck pain questionnaire, and problem elicitation technique for measuring disability associated with whiplash-associated disorders. Pain 2003, 102, 273-281. [CrossRef]

64. Kasch, H.; Bach, F.W.; Jensen, T.S. Handicap after acute whiplash injury: A 1-year prospective study of risk factors. Neurology 2001, 56, 1637-1643. [CrossRef]

65. Kasch, H.; Qerama, E.; Kongsted, A.; Bach, F.W.; Bendix, T.; Jensen, T.S. The risk assessment score in acute whiplash injury predicts outcome and reflects biopsychosocial factors. Spine 2011, 36, S263-S267. [CrossRef]

66. Kasch, H.; Jensen, L.L. Minor Head Injury Symptoms and Recovery From Whiplash Injury: A 1-Year Prospective Study. Rehabil. Process. Outcome 2019, 8, 1-6. [CrossRef]

67. Krogh, S.; Kasch, H. Whiplash injury results in sustained impairments of cervical muscle function: A one-year prospective, controlled study. J. Rehabil. Med. 2018, 50, 548-555. [CrossRef]

68. Leth-Petersen, S.; Rotger, G.P. Long-term labour-market performance of whiplash claimants. J. Health Econ. 2009, 28 , 996-1011. [CrossRef]

69. Mankovsky-Arnold, T.; Wideman, T.H.; Thibault, P.; Lariviere, C.; Rainville, P.; Sullivan, M.J.L. Sensitivity to Movement-Evoked Pain and Multi-Site Pain are Associated with Work-Disability Following Whiplash Injury: A Cross-Sectional Study. J. Occup. Rehabil. 2017, 27, 413-421. [CrossRef]

70. Munjin, M.A.; Zamorano, J.J.; Marre, B.; Ilabaca, F.; Ballesteros, V.; Martinez, C.; Yurac, R.; Urzua, A.; Lecaros, M.; Fleiderman, J.; et al. Speed hump spine fractures: Injury mechanism and case series. J. Spinal. Disord Tech. 2011, 24, 386-389. [CrossRef] 
71. Myrtveit, S.M.; Carstensen, T.; Kasch, H.; Ornbol, E.; Frostholm, L. Initial healthcare and coping preferences are associated with outcome 1 year after whiplash trauma: A multicentre 1-year follow-up study. BMJ Open 2015, 5, e007239. [CrossRef]

72. O'Hara, N.N.; Mugarura, R.; Potter, J.; Stephens, T.; Rehavi, M.M.; Francois, P.; Blachut, P.A.; O’Brien, P.J.; Mezei, A.; Beyeza, T.; et al. The Socioeconomic Implications of Isolated Tibial and Femoral Fractures from Road Traffic Injuries in Uganda. J. Bone Joint Surg. Am. 2018, 100, e43. [CrossRef]

73. Pieske, O.; Weinhold, T.; Buck, J.; Piltz, S. Seniority of the first-treating doctor does not influence the outcome of acute whiplash injury: A prospective cohort study. Eur. Spine J. 2010, 19, 1627-1634. [CrossRef]

74. Prang, K.H.; Berecki-Gisolf, J.; Newnam, S. Recovery from musculoskeletal injury: The role of social support following a transport accident. Health Q. Life Outcomes 2015, 13, 97. [CrossRef]

75. Ratzon, N.Z.; Amit, Y.; Friedman, S.; Zamir, S.; Rand, D. Functional capacity evaluation: Does it change the determination of the degree of work disability? Disabil. Health J. 2015, 8, 80-85. [CrossRef]

76. Rebbeck, T.; Sindhusake, D.; Cameron, I.D.; Rubin, G.; Feyer, A.M.; Walsh, J.; Gold, M.; Schofield, W.N. A prospective cohort study of health outcomes following whiplash associated disorders in an Australian population. Inj. Prev. 2006, 12, 93-98. [CrossRef]

77. Rosenthal, R.E.; Coker, W.L. Posterior fracture-dislocation of the hip: An epidemiologic review. J. Traum. 1979, 19, 572-581. [CrossRef]

78. Sarrami, P.; Ekmejian, R.; Naylor, J.M.; Descallar, J.; Chatterji, R.; Harris, I.A. Spine surgery outcome in patients who sought compensation after a motor vehicle accident: A retrospective cohort study. BMC Surg. 2016, 16, 76. [CrossRef]

79. Schreiber, A.L.; Fried, G.W. Demographic characteristics of 38 patients injured in motor vehicle accidents referred by chiropractors to physiatrists. J. Manip. Physiol. Ther. 2009, 32, 772-775. [CrossRef]

80. Scuderi, G.J.; Sherman, A.L.; Brusovanik, G.V.; Pahl, M.A.; Vaccaro, A.R. Symptomatic cervical disc herniation following a motor vehicle collision: Return to work comparative study of workers' compensation versus personal injury insurance status. Spine J. 2005, 5, 639-644. [CrossRef]

81. Smed, A. Cognitive function and distress after common whiplash injury. Acta. Neurol. Scand 1997, 95, 73-80. [CrossRef]

82. Swartzman, L.C.; Teasell, R.W.; Shapiro, A.P.; McDermid, A.J. The effect of litigation status on adjustment to whiplash injury. Spine 1996, 21, 53-58. [CrossRef]

83. Virani, S.N.; Ferrari, R.; Russell, A.S. Physician resistance to the late whiplash syndrome. J. Rheumatol. 2001, 28, $2096-2099$.

84. Vos, C.J.; Verhagen, A.P.; Passchier, J.; Koes, B.W. Impact of motor vehicle accidents on neck pain and disability in general practice. Br. J. Gen. Pract. 2008, 58, 624-629. [CrossRef]

85. Chen, K.; Andersen, T.; Carroll, L.; Connelly, L.; Côté, P.; Curatolo, M.; Elliott, J.; Grant, G.; Jull, G.; Kasch, H.; et al. Recommendations For Core Outcome Domain Set For Whiplash-Associated Disorders (CATWAD). Clin. J. Pain 2019, 35, 727-736. [CrossRef]

86. Kelly, J.; Ritchie, C.; Sterling, M. Clinical prediction rules for prognosis and treatment prescription in neck pain: A systematic review. Musculoskelet. Sci. Pract. 2017, 27, 155-164. [CrossRef]

87. Wong, J.J.; Côté, P.; Shearer, H.M.; Carroll, L.J.; Yu, H.; Varatharajan, S.; Southerst, D.; van der Velde, G.; Jacobs, C.; Taylor-Vaisey, A. Clinical practice guidelines for the management of conditions related to traffic collisions: A systematic review by the OPTIMa Collaboration. Disabil. Rehabil. 2015, 37, 471-489. [CrossRef] [PubMed]

88. United Nations. World Economic Situation and Prospects; United Nations Department of Economic and Social Affairs: New York, NY, USA, 2020.

89. Bhalla, K.; Shotten, M.; Cohen, A.; Brauer, M.; Shahraz, S.; Burnett, R.; Leach-Kemon, K.; Freedman, G.; Murray, C.J.L. Transport for Health: The Global Burden of Disease from Motorized Road Transport; World Bank Group: Washington, DC, USA, 2014.

90. Marnetoft, S.-U. Vocational Rehabiliation. In Handbook of Vocational Rehabilitation and Disability Evaluation; Escorpizo, R., Brage, S., Homa, D., Stucki, G., Eds.; Spinger: Cham, Switzerland, 2015; pp. 73-103. [CrossRef]

91. World Health Organization. Global Lan for the Decade of Action for Road Safety 2021-2030; WHO: Geneva, Switzerland, 2021.

92. International Road Foundation. HOVITA Launches the Motor Third Party Insurance Compensation Project for Uganda's Road Crash Victims. Available online: https:/ /irfnet.ch/2020/11/30/hovita-launches-the-motor-third-partyinsurance-compensationproject-for-ugandas-road-crash-victims/ (accessed on 22 October 2021).

93. Gosselin, R.A.; Spiegel, D.A.; Coughlin, R.; Zirkle, L.G. Injuries: The neglected burden in developing countries. Bull. World Health Organ. 2009, 87, 246. [CrossRef] [PubMed]

94. Bevan, S. Economic impact of musculoskeletal disorders (MSDs) on work in Europe. Best Pract. Res. Clin. Rheumatol. 2015, 29, 356-373. [CrossRef] [PubMed]

95. von Oelreich, E.; Eriksson, M.; Brattström, O.; Discacciati, A.; Strömmer, L.; Oldner, A.; Larsson, E. Predicting prolonged sick leave among trauma survivors. Sci. Rep. 2019, 9, 58. [CrossRef] [PubMed]

96. Himmelstein, D.U.; Warren, E.; Thorne, D.; Woolhandler, S. Illness And Injury As Contributors To Bankruptcy. Health Aff. 2005, 24, W5-63-W65-73. [CrossRef]

97. DeRigne, L.; Stoddard-Dare, P.; Quinn, L. Workers Without Paid Sick Leave Less Likely To Take Time Off For Illness Or Injury Compared To Those With Paid Sick Leave. Health Aff. 2016, 35, 520-527. [CrossRef]

98. International Labour Organization. Non-Standard Employment around the World: Understanding Challenged, Shaping Prospects; ILO: Geneva, Switzerland, 2016.

99. Transport Accident Commission. Income Support. Available online: http://www.tac.vic.gov.au/clients/how-we-can-help/ income-support (accessed on 22 October 2021). 
100. Motor Accident Insurance Commission. What You Can Claim. Available online: https://maic.qld.gov.au/for-injured-people/ what-you-can-claim/ (accessed on 22 October 2021).

101. Samoborec, S.; Ruseckaite, R.; Ayton, D.; Evans, S. Biopsychosocial factors associated with non-recovery after a minor transportrelated injury: A systematic review. PLoS ONE 2018, 13, e0198352. [CrossRef]

102. Smits, E.J.; Gane, E.M.; Brakenridge, C.L.; Andrews, N.E.; Johnston, V. Expert consensus and perspectives on recovery following road traffic crashes: A Delphi study. Disabil. Rehabil. 2020, 1-10. [CrossRef] 